\title{
Optical and Structural Characterization of GaN Based Hybrid Structures and Nanorods
}

\author{
Mathias Forsberg
}

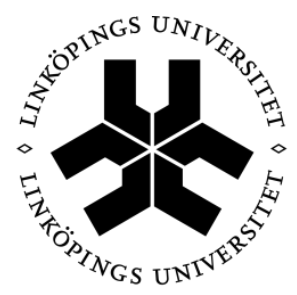

\section{Linköping University}

INSTITUTE OF TECHNOLOGY

Thin Film Physics Division

Department of Physics, Chemistry, and Biology (IFM)

Linköping University

SE-581 83 Linköping, Sweden 
Linköping Studies in Science and Technology

Thesis No. 1703

Author:

Mathias Forsberg

Thin Film Physics Division

Department of Physics, Chemistry, and Biology (IFM)

Linköping University

SE-581 83 Linköping, Sweden

matfo@ifm.liu.se

Copyright (c) Mathias Forsberg, unless otherwise stated.

All rights reserved

Mathias Forsberg

Optical and Structural Characterization of

GaN Based Hybrid Structures and Nanorods

ISBN: 978-91-7519-141-6

ISSN: 0280-7971

Printed by LiU Tryck, Linköping, 2015 


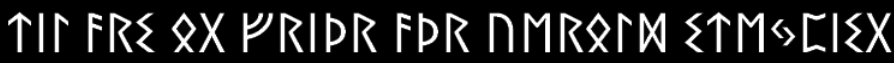

'To a prosperous year and tranquility until the world falls' 


\section{Abstract}

$\mathrm{GaN}$ belongs to the group III nitrides and is today the material of choice for efficient blue light emission, enabling solid state white lighting by combining red, blue and green light emitting diodes (LED) or by having a blue LED illuminating a phosphor. By combining GaN quantum well (QW) structures with colloids, nanoparticles or polyfluorene films, LEDs may be fabricate at lower cost. Such hybrid structures are promising for future micro-light sources in full-color displays, sensors and imaging systems. In this work, hybrid structures based on an MOCVD grown GaN QW sandwiched between two layers of AlGaN have been studied. On top of the structure, colloidal $\mathrm{ZnO}$ nano-crystals were deposited by spin-coating. Time-resolved photoluminescence was used to investigate the $\mathrm{QW}$ exciton dynamics in these hybrids depending on the cap layer thickness. From comparison of the recombination rate in the bare QW structure and the hybrid, the efficiency of the non-radiative resonant energy transfer between the QW and the nano-crystals could be obtained.

Bulk GaN of large area is difficult to synthesize. Thus, due to lack of native substrates, GaN-based structures are grown on $\mathrm{SiC}$ or sapphire, which results in high threading dislocation density in the active layer of the device. Fabricating GaN nanorods (NR) can be a way to produce GaN with lower defect density since threading dislocations are annihilated toward the NR wall during growth. Here, $\mathrm{GaN}(0001)$ NRs grown on $\mathrm{Si}(111)$ substrates by magnetron sputter epitaxy using a liquid Ga target have been investigated. Sputter deposition has the advantage of being easy to scale up for depositions on large surfaces. It is also possible to deposit at lower temperatures, which allows the use of substrates with lower decomposition temperature. In the second paper of this thesis, optical and structural properties of sputtered GaN NRs have been studied. 


\section{Preface}

This licentiate thesis summarizes my work at Linköping University at the Department of Physics, Chemistry and Biology (IFM) within the Thin Film Physics division from May 2012 to January 2015. The aim of this thesis is to study optical and structural properties of GaN based nano structures. I believe that hybrid structures based on $\mathrm{GaN}$ can have a great potential for photonics and optoelectronics and should be a subject for further investigation in the future. The project was financed by the Swedish Energy Agency and CeNano and I appreciate this support. 


\section{Acknowledgment}

I would like to thank everyone who helped me with my licentiate studies at LiU, in particular:

My supervisor Associate Professor Galia Pozina: Thank you very much for your excellent supervision and support in my progress. I highly admire your skill and scientific knowledge within the field and I appreciate your dedication to help me.

My co-supervisor Dr. Ching-Lien Hsiao: Thank you for all your help over the years, especially in teaching me the deposition systems which I am forever grateful of.

Professor Jens Birch: I am very thankful for being a part of the thin film division and you are a good role model for every student here.

Co-authors; Alexandra Serban, Iuliana Poenaru, Dr. Mohammad Junaid, Docent Carl Hemmingsson and Professor Hiroshi Amano: Thank you, it has been a pleasure!

Thomas Lingefeldt and Harri Savimäki: Thank you for all the times you have fixed the equipment and instruments making my experiments and characterizations run smoothly.

To all the others who helped me understand and fix the deposition systems; Dr. Agne Žukauskaité and Dr. Per Sandström, thank you very much.

Min familj; Patric, Gunnel och Andreas Forsberg: Tack för ert stöd och att ni matade min nyfikenhet sedan innan den första skoldagen. 


\section{Included Papers}

I. Dynamic properties of excitons in $\mathrm{ZnO} / \mathrm{AlGaN} / \mathrm{GaN}$ hybrid nanostructures

Mathias Forsberg, Carl Hemmingsson, Hiroshi Amano and Galia Pozina

Scientific reports 5:7889 (2015)

II. Stacking fault related luminescence in GaN nanorods

Mathias Forsberg, Alexandra Serban, Iuliana Poenaru,

Mohammad Junaid, Ching-Lien Hsiao, Jens Birch and Galia

Pozina

Manuscript in preparation 


\section{Contents}

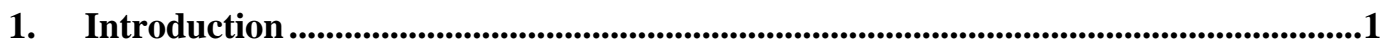

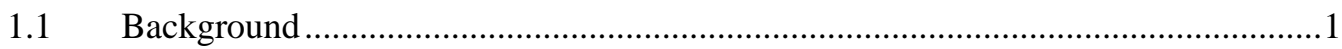

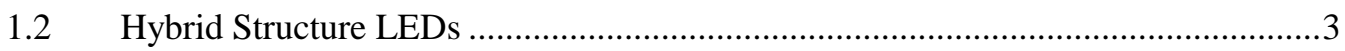

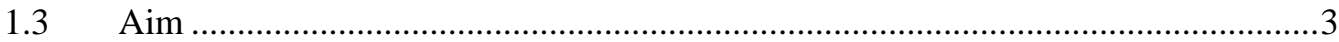

1. Group III-Nitrides...............................................................................................................................5

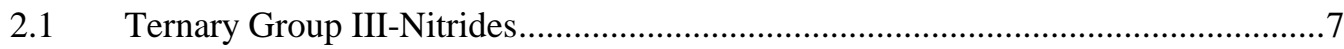

2. Excitons and Band Structures in Semiconductors ............................................................9

3.1 Förster Resonance Energy Transfer ....................................................................13

3. Introduction to Vacuum Theory ....................................................................................................17

5. Introduction to Sputter Deposition Theory..........................................................................21

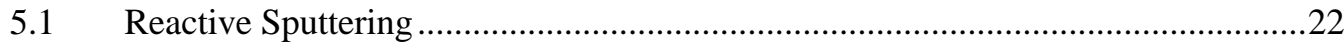

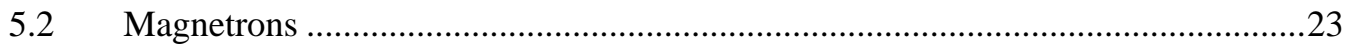

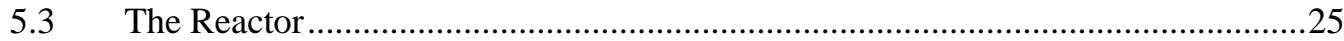

6. Metal-Organic Chemical Vapor Deposition............................................................................27

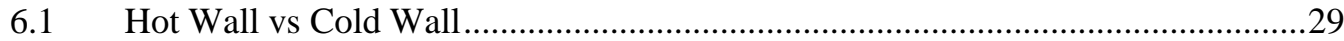

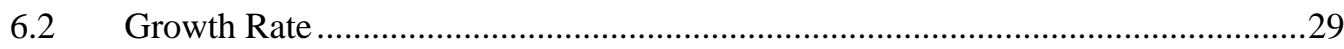

7. Characterization ...................................................................................................................31

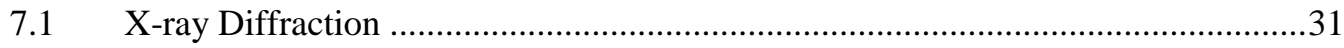

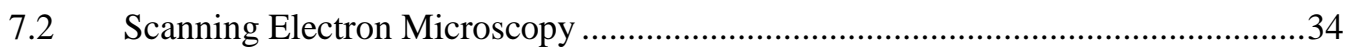

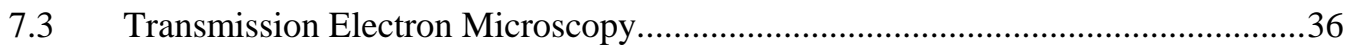

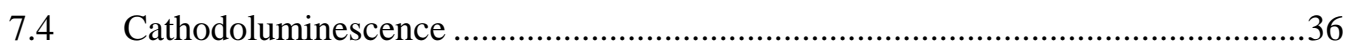

7.5 Photoluminescence and Time-Resolved Photoluminescence ...................................37

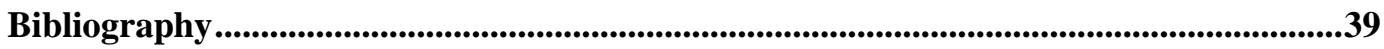

8. Summary of Included Papers ............................................................................................45 



\section{Introduction}

\subsection{Background}

$\mathrm{GaN}$ is a semiconductor which belongs to the group III-nitrides. GaN, together with its alloys of $\mathrm{Al}$ and $\mathrm{In}$ are of high interest at present. The main public focus is related to their light emitting properties which are important for such applications as UV and blue LEDs. In this connection it is worth to mention that the Nobel Prize 2014 in physics was awarded to Isamu Akasaki, Hiroshi Amano and Shuji Nakamura for their work on $\mathrm{GaN}$ based blue LED. Additionally, $\mathrm{GaN}$ also has excellent electrical properties making it suitable for e.g. high frequency and high power devices ${ }^{1-3}$.

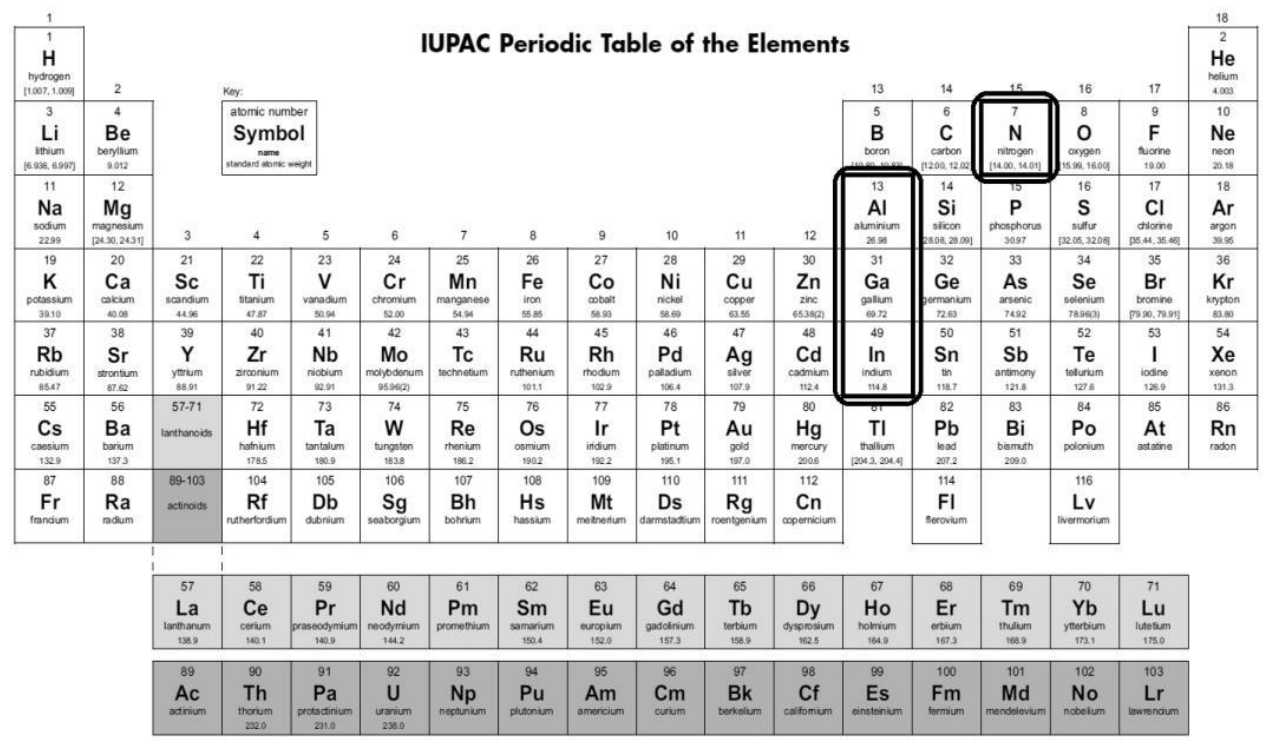

Figure 1. Official IUPAC periodic table ${ }^{4}$ with aluminum, gallium and indium of group III and nitrogen of group $\mathrm{V}$ highlighted 
GaN in industrial scale cannot be synthesized from a liquid phase ${ }^{5}$. Therefore, different methods have been developed to grow $\mathrm{GaN}$ from a vapor phase ${ }^{6,7}$. To this date, only few studies have considered sputtered $\mathrm{GaN}$ from a liquid gallium target with nitrogen as working gas ${ }^{8}$. An alternative way to produce $\mathrm{GaN}$ by sputtering is to sputter $\mathrm{Ga}_{2} \mathrm{O}_{3}$ followed by ammonization ${ }^{9,10}$.

Also, the lack of low cost high quality GaN substrates of large areas requires commercial devices to be grown on e.g. $\mathrm{Si}, \mathrm{SiC}$ or sapphire. Due to the huge lattice miss-match between $\mathrm{GaN}$ and the substrate materials, defects such as dislocations are introduced in the film. The dislocation density can be up to $10^{11}-10^{12} \mathrm{~cm}^{-2}$, which lowers the efficiency of the device. Akasaki and Amano, besides the invention of efficient p-type doping in $\mathrm{GaN}$, have also found that by employing an additional buffer layer (AlN, or low temperature $\mathrm{GaN}$ ) on sapphire before depositing the GaN basestructure, the defect density in the active layer can be significantly reduced ${ }^{11,12}$. After this discovery, the explosive commercialization of $\mathrm{GaN}$ based devices could be started. However, the dislocation density is still high and a common defect in GaN thin films today, which are affecting the device performance, are threading dislocations ${ }^{13,14}$. By growing GaN nanorods (NR), threading dislocations can be annihilated at the NR sides $^{15,16}$. This give rise to GaN structures with high crystal quality. Additionally, these types of structures can be grown on substrates with even higher lattice mismatch which enables the use of the mature fabrication processes of cheaper substrates, e.g. Si.

This work is focused on luminescence properties of GaN-based structures. A valence electron in a semiconductor can, if given enough energy, become excited. It is no longer restrained to the bonds in the crystal, but is now free to move across the lattice, i.e. is in the conduction band. As the electron has this new energy state, it also leaves the "absence of an electron" in the valence band, which is called a hole. The electron and hole can form a bound state (particle called exciton) due to an attractive Coulomb's force between them. An exciton can then recombine, i.e. the electron return to its previous energy state. The excess energy it once possessed needs to be released. The recombination process can be radiative, with emission of light or non-radiative, by dissipation of phonons, i.e. by producing heat. The non-radiative recombination can also be caused by different impurities or defects having additional energy levels within the band gap. For LEDs, the radiative recombination is a demand and that is why a high crystal quality is important. 


\subsection{Hybrid Structure LEDs}

If the emission spectrum of one semiconductor is overlapping with the absorption spectrum of another semiconductor, the energy of the photon emitted can be used to radiatively pump the second semiconductor. This principle is used in hybrid LEDs (HLED) ${ }^{17-19}$ for efficient down conversion of UV light to visible light. This is especially important for the green region, where there is still lack of efficient LEDs. HLED structures are fabricated using a GaN LED and a layer of fluorescent material such as an organic polyfluorene or colloid nanocrystals, thus combining the advantages of both components. Organics can be engineered to emit light of different colors based on functional groups or by mixing a blend of several polymers ${ }^{20}$. Different coloremitting colloids can also easily be mixed. However, organics have very low carrier mobility and electrical pumping is therefore inefficient to obtain electroluminescence ${ }^{21}$. It is also difficult to make electrical contacts on nanocrystals, and, thus to use them directly for fabrication of commercial LEDs. The higher carrier mobility of the inorganics makes them more suitable to electrical contacts ${ }^{22}$. That is why one could utilize the electrically pumped efficient inorganic GaN-based LED to radiatively pump the polymer/colloidal film with fluorescence in the visible region.

Recently, a novel class of HLED structures has been suggested. As described by T. Förster in the late 1940's and 50's ${ }^{23-25}$, Energy transfer between an energy donor and an energy acceptor material is possible if the spectral overlap is wide and the spatial distance between them is small. This phenomenon is discussed in Paper I and more details can be found in Chapter 3 .

\subsection{Aim}

The aim of this thesis is to study the optical and structural properties of GaN nano-structures (quantum wells and NRs) to further understand radiative mechanisms responsible for the near band gap luminescence. Hybrid structures based on $\mathrm{AlGaN} / \mathrm{GaN}$ quantum wells and $\mathrm{ZnO}$ nanocrystals were studied in Paper I and GaN NRs in Paper II. 


\section{Group III-Nitrides}

Group III-nitrides can crystallize in either wurtzite or zinkblende structure. The wurtzite structure is thermodynamically stable (for all binary, ternary and quaternary alloys). This crystal structure is therefore the easiest to grow and most studies, including this thesis, are done on the wurtzite structured nitrides ${ }^{26,27}$. In the wurtzite structure the atoms are placed in the lattice points of two interpenetrating hexagonal close packed lattices. The lattices will be relatively shifted by $\frac{3}{8}$ the height of the lattice cell ${ }^{28}$ as illustrated in figure 2. Table 1 below summarized the conventional way of naming planes and directions in terms of Miller indices. The bonds are polar covalent and each atomic species is placed in tetrahedral coordination due to the $\mathrm{sp}^{3}$ hybridization of the bonds.

[0001]

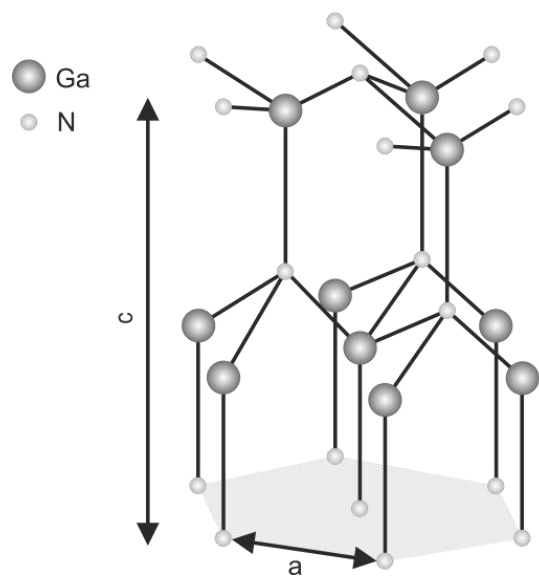

[0001]

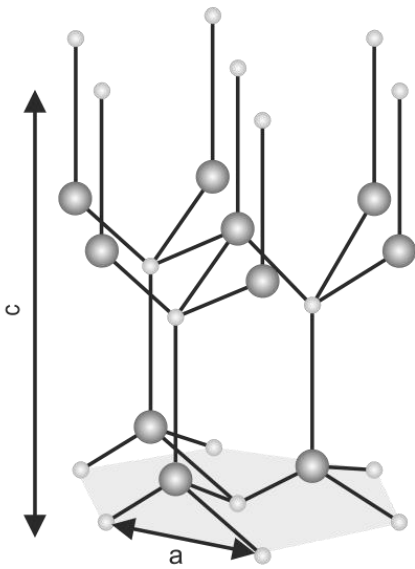

Figure 2. Illustration of the wurtzite structure. Notice the lack of inversion symmetry as the structure is flipped along the c-axis

A wurtzite structure does not have inversion symmetry, i.e. the direction [0001] and $[000 \overline{1}]$ is not equivalent. [0001] is usually called positive c-direction while [000 $\overline{1}]$ is usually called negative c-direction. If a $\mathrm{GaN}$ crystal is rotated $180^{\circ}$ while in [0001] direction, $\mathrm{Ga}$ and $\mathrm{N}$ will exchange positions with each other. 
Table 1. Crystallographic conventions for naming planes and directions in a lattice.

\begin{tabular}{l|l|} 
Nomenclature & Meaning \\
\hline$[h k l]$ & One specific direction \\
$(h k l)$ & One specific plane \\
$\{h k l\}$ & Crystallographically equivalent planes \\
$\langle h k l\rangle$ & Crystallographically equivalent directions \\
\cline { 2 - 2 } &
\end{tabular}

The wurtzite structure exhibits a macroscopic polarization along the c-axis even in the absence of strain. The presence of lattice mismatch in heterostructures results in an additional contribution to the total polarization due to the piezoelectric effect. This is detrimental to LED technologies ${ }^{29}$ where a very thin film is sandwiched between materials with higher band gap forming a so called quantum well structure. In III-N heterostructures grown in c-direction, the conduction and valence bands in the quantum well and in the barrier layers will be bent by this polarization, thus separating the charge carriers and consequently decreasing the probability of radiative recombination.

The interest in group III-Ns is caused by their spectacular optical and electrical properties making them suitable for different optoelectronic and electronic applications. All group III-Ns are direct band gap semiconductors. A semiconductor with a direct band gap has the maximum of the valence band and the minimum of the conduction band at the same point in reciprocal space as will be discussed in Chapter 3. This is different from indirect band gap semiconductors where electrons at the minimum of the conduction band and the maximum of the valence band do not have the same momentum and therefore recombination processes involve the emission or absorption of phonons to achieve conservation of the momentum when an electron recombines with a hole. Table 2 summarizes lattice parameters and band gap energies of wurtzite AlN, $\mathrm{GaN}$ and InN.

Table 2. Collected parameters for wurtzite structured group III-nitrides. For more parameters see reference ${ }^{30}$.

\begin{tabular}{l|lll|} 
Parameter & AlN & GaN & InN \\
\hline Lattice parameter $\boldsymbol{a}(T=300 \mathrm{~K})[\AA]$ & 3.112 & 3.189 & 3.545 \\
Lattice parameter $\boldsymbol{c}(T=300 \mathrm{~K})[\AA]$ & 4.982 & 5.185 & 5.703 \\
Band gap $\boldsymbol{E}_{\boldsymbol{g}}[\mathrm{eV}]$ & 6.25 & 3.510 & 0.78 \\
\cline { 4 - 4 }
\end{tabular}




\subsection{Ternary Group III-Nitrides}

The ternary III-Ns, $A_{x} B_{1-x} N$, alloys can be carefully engineered in respect to composition. The behavior of lattice parameters, $a$ in meters, and energy band gaps, $E_{g}$ in electron volts, with the change of the composition can be described by Vegard's law (equation 1 and 2 respectively).

$$
\begin{aligned}
& a\left(\mathrm{~A}_{\mathrm{x}} \mathrm{B}_{1-\mathrm{x}} \mathrm{N}\right)=\mathrm{x} \cdot a_{\mathrm{A}}+(1-\mathrm{x}) \cdot a_{\mathrm{B}} \\
& E_{g_{\mathrm{A}_{\mathrm{x}} \mathrm{B}_{1-\mathrm{x}} \mathrm{N}}}=\mathrm{x} \cdot \mathrm{E}_{\mathrm{g}_{\mathrm{A}}}+(1-\mathrm{x}) \cdot \mathrm{E}_{\mathrm{g}_{\mathrm{B}}}+\mathrm{b} \cdot \mathrm{x} \cdot(1-\mathrm{x})
\end{aligned}
$$

Where $b$ is the bowing parameter in electron volts. Values for the band gap energies and the lattice constants as well as the bowing parameter were taken from Ref. ${ }^{30}$ and used to plot figure 3. A comprehensive collection of parameters of III-V alloys have been published by the same group ${ }^{31}$. With energy band gaps between $0.78 \mathrm{eV}$ and $6.25 \mathrm{eV}$ and lattice parameters, $a$, from $3.112 \AA$ and $3.545 \AA$ the Al-Ga-In-N alloys offer a broad spectrum of engineering possibilities. From analyzing figure 3 some interesting results can be deducted. For example, AlInN can be made lattice matched to $\mathrm{GaN}$ while having a wider band gap.

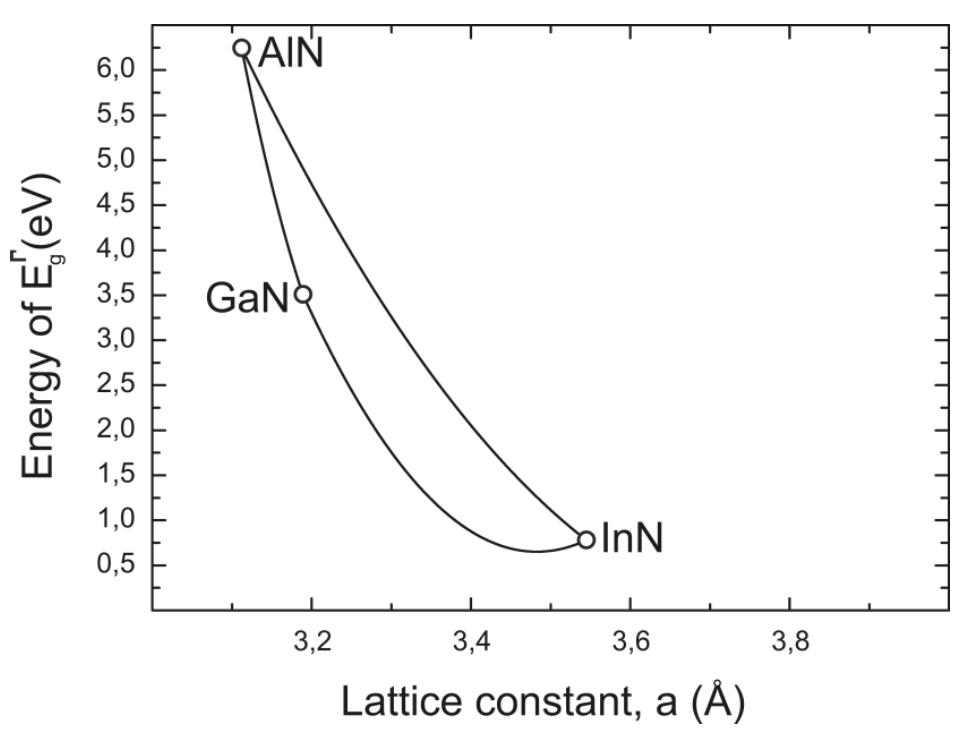

Figure 3. Band gap energy in $\Gamma$ point vs lattice constant a plotted using the data obtained from I. Vurgaftman and J. R. Meyer ${ }^{30}$ 


\section{Excitons and Band Structures in Semiconductors}

When a valence electron is moving across the crystal structure it experiences a periodic potential due to the nuclei. To find the energy of a particle moving in a periodic potential we use Schrödinger's equation.

$$
\left(-\frac{\hbar^{2}}{2 m} \nabla^{2}+U(r)\right) \Psi=E \Psi
$$

Where $\hbar$ is the reduced Planck's constant $\left(1.054571726 \cdot 10^{-34} \mathrm{~J} / \mathrm{s}\right), m$ is the electron mass in kilograms, $U(r)$ is the potential in Joule, $\Psi$ is the wave function describing the electron and $E$ is the energy of the electron in Joule. The solution to equation 3 for a free electron (in an isotropic material) gives the energy dispersion of the electron:

$$
E=\frac{\hbar^{2}}{2 m} \mathrm{k}^{2}
$$

Where $\mathrm{k}$ is an arbitrary reciprocal space vector in reciprocal meters. The energy dependence on $\mathrm{k}$ is illustrated in figure 4 . The area within the parabolas reflects the energy states of the electrons. The bottom parabola gives solutions to the Schrödinger equation related to the filled states, i.e. the states occupied by the electrons. The top parabola relates to the solutions of the empty states. In semiconductors and insulators there is a gap between them. This gap is the region of energy for which, at any k-values, there are no existing solutions to the Schrödinger equation. This difference in the energy between the bottom of the conduction band and the top of the valence band is called the band gap. 


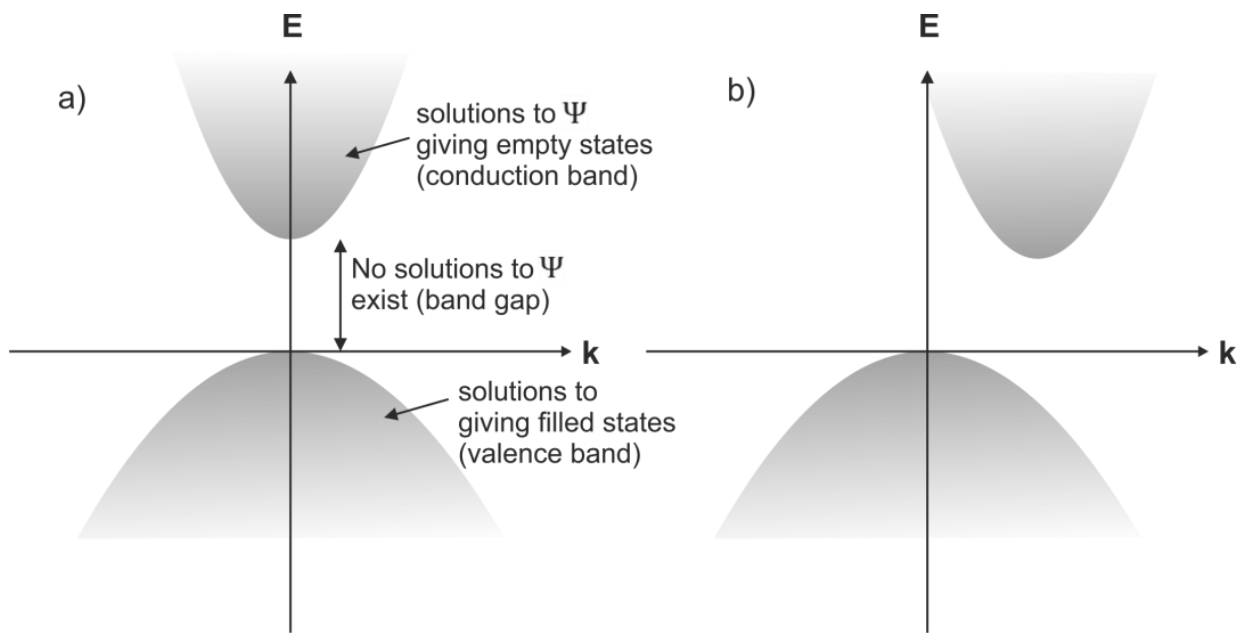

Figure 4. Schematic drawing of a band structure in semiconductors: a) Illustrates a direct bandgap while b) illustrates an indirect bandgap. The energy dispersion is plotted vs $\mathrm{k}$. Bottom and upper parabolas reflect the filled and empty electron energy states, respectively. In semiconductors and insulators an energy gap is present where no electron states, $\boldsymbol{\Psi}$, exist.

If the top of the valence band and the bottom of the conduction band is at the same point of the reciprocal lattice (as in figure 4a) the band gap is called a direct band gap. If, as shown in figure $4 \mathrm{~b}$, they do not align in reciprocal space, it is called an indirect band gap. Electron transitions between each band must adhere to the conservation of energy and momentum. Thus in the indirect band gap semiconductor the transition will be phonon-assisted to fulfill this requirement. Phonon-assisted transitions have low probability (as involving three particles) and that is why for fabrication of the efficient LEDs it is important to use direct band gap materials. For example, SiC, which is an indirect band gap semiconductor, has similar band gap energies as GaN; However, it could not be used to produce a blue LED of sufficient efficiency ${ }^{32}$.

The dispersion relation only shows how the energy depends on the $\mathrm{k}$ vector. To know how many states there are within a given energy one use a concept of density of states, DOS, or $\mathrm{D}(\mathrm{E})$. The energy states available for electrons and holes are restricted by dimensionallity as illustrated in figure 5 . In the top of figure 5 there are schematic drawings showing the energy dependence of DOS in the conduction band for different cases. As energy is decreasing one can see how the DOS is approaching zero. This corresponds to the top of the band gap illustrated in figure 4 . 

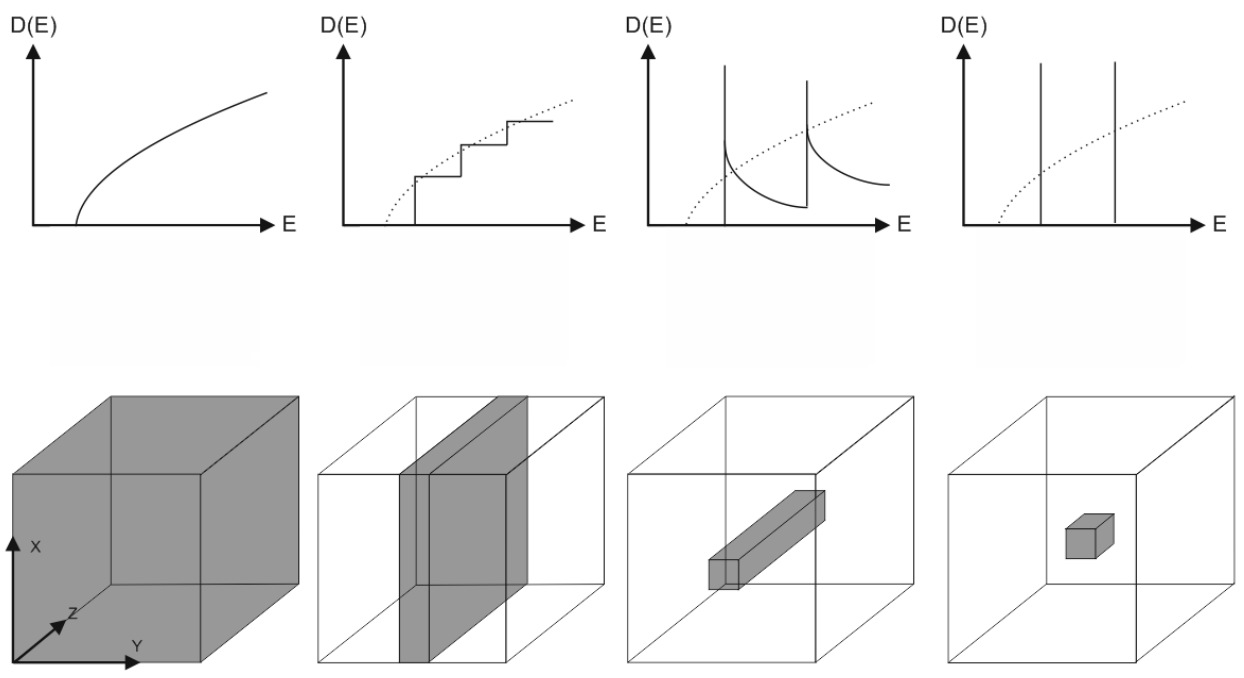

Figure 5. Schematic illustration of how dimensionality of the crystal affects the density of state. From left; 3D, 2D, 1D, 0D cases corresponding to bulk, quantum well, quantum rod and quantum dot respectively. The schematic drawings of DOS at the top illustrates the conduction band DOS for each case.

In metals the valence band and conduction band overlaps. The valence electrons are free to move across the lattice, this is what allows electric current to flow through the metal. In insulators and semiconductors the valence electrons are "static" forming bonds with the surrounding atoms. The band gap in diagrams shown in figure 4 and 5 is representing the required energy, which is needed for a valence electron to break from the bonds and to become a conducting electron, which can move free across the lattice in similar ways as in metals.

When electrons are excited to higher energy states they leave "the absence" of an electron, i.e. a hole in the valence band. If the electron doesn't get enough energy to escape the hole it can be pulled back in by the Coulomb's force and recombine to emit light or become an exciton. Excitons are electron-hole pairs bound by an attractive Coulomb's force, and thereby form an electrically neutral quasi-particle. Excitons are usually separated into two types based on their Bohr radius; the Frenkel exciton (exciton with small Bohr radius) and the Wannier-Mott exciton (having a large Bohr radius). The Coulomb's force, $F$, can be written as:

$$
F=\frac{Q_{1} Q_{2}}{4 \pi \varepsilon_{0} \varepsilon_{r} r^{2}}
$$


Where $Q_{1}$ and $Q_{2}$ are the carrier charges separated by a distance $r$ and $\varepsilon_{r}$ is the dielectric constant of the material. The attractive force between the two charges decreases if $\varepsilon_{r}$ increases. The decreased forced also means that the Bohr radius increases. The Frenkel excitons are therefore found in polyfluorenes (where $\varepsilon$ is below $\sim 4$ ) ${ }^{33}$ while in the group III-nitrides $(\varepsilon \text { is } 5-20)^{34}$ the Wannier-Mott excitons will be formed. Exciton binding energies are on the order of $0.1-1 \mathrm{eV}$ and $0.01-0.1 \mathrm{eV}$ for Frenkel and WannierMott respectively and the Bohr radius are on the order of a few $\mathrm{nm}$ to 10 's of $\mathrm{nm}$ for Frenkel to Wannier-Mott, respectively. These two types of excitons have different properties. The Wannier-Mott excitons start to interact at much lower densities in the material because their wave functions are more spread. This allows for optical nonlinearity due to many-particle effects ${ }^{35}$.

The luminescence emitted from the sample following a radiative recombination has different names, e.g.; cathodoluminescence, electroluminescence and photoluminescence depending on the source that provided the energy for the excitation of the electron, which is electron bombardment, electric field and electromagnetic radiation, respectively.

Figure 6 illustrates several possible recombination processes. All transitions shown are radiative except $b$ ) which is the relaxation of the excited electron (or hole) to the conduction band (valence band) minimum (maximum).

Upon free carrier recombination the emitting photons have higher energies than upon exciton recombination (as shown in figure $6 \mathrm{c}, \mathrm{d}$ ) because of the binding energy of the exciton. In bulk GaN the binding energy is about $20 \mathrm{meV}$ while it is slightly increased to $28-35 \mathrm{meV}$ in quantum well structures according to theoretical predictions ${ }^{36}$. The difference can be understood in terms of the exciton energy being determined not only by the Coulomb potential, but also by the carrier confinement in a potential well ( i.e. the space the carriers are allowed in the growth direction is limited by the thickness of the quantum well). If the space allowed for the carriers are on the order of the exciton Bohr radius then the exciton is quantum confined. 


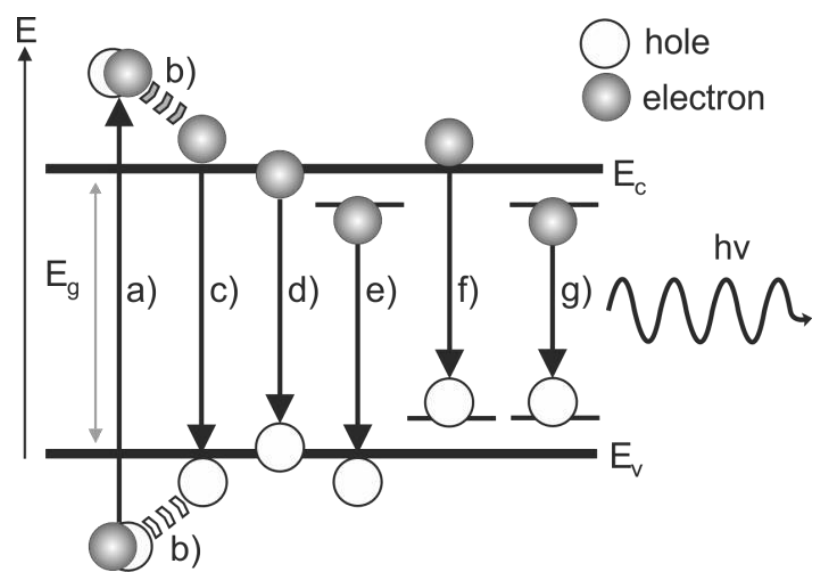

Figure 6. Schematic drawing of optical transitions in a semiconductor. Different processes are shown; a) the excitation of an electron from within the valence band to the conduction band; b) the nonradiative relaxation of the electron and hole down to the respective band edge; c) the free carrier radiative recombination; d) the free exciton radiative recombination; e) the donor bound exciton recombination; f) the acceptor bound recombination; g) the donor-acceptor pair recombination.

\subsection{Förster Resonance Energy Transfer}

Förster (or fluorescence) resonance energy transfer (FRET) was briefly discussed in the Introduction. Here is a deeper inquiry of the phenomenon. As aforementioned, excitons can be described as electrically neutral quasi-particles. However, excitons consist of two spatially separated charges with opposite sign (i.e. they're dipoles). Thus, excitons have a dipole moment. In the presence of two closely spaced materials (so called energy donor and energy acceptor), it is possible that an energy transfer (i.e. FRET) occur between two dipoles due to dipole-dipole coupling ${ }^{37}$, see figure 7 .

$$
D^{*}+A \rightarrow A^{*}+D
$$

Here, $\mathrm{D}$ is the energy donor and $\mathrm{A}$ is the energy acceptor. The asterisk denotes which of the donor or acceptor that is excited. It is important to mention that FRET is a nonradiative energy transfer. The acceptor dipole does not gain the energy through the absorption of a photon. It was first published by Theodor Von Förster ${ }^{23-25}$ and the theory was originally developed to study the equilibrium and dynamical properties of e.g. organic compounds or biopolymers. 
a)

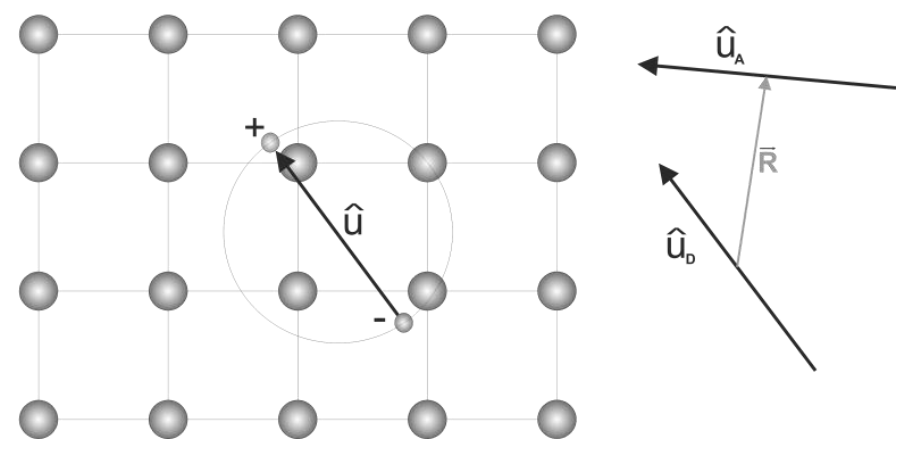

Figure 7. Illustration of a) the origin of dipole moment $\hat{\boldsymbol{u}}$ in an exciton. b) Is illustrating an arbitrary donor and acceptor dipole moment alignment at a distance $\mathrm{R}$. If $\mathrm{R}$ is small, dipole-dipole coupling can occur.

If the donor excited state is resonant with the band gap of the acceptor and the spatial distance between the excitons is small, the energy needed to excite the acceptor dipole can be transferred.

When choosing compatible energy donor and acceptor materials for fabrication of so called hybrid structures, the spectral emission of the donor should overlap widely with the absorption of the acceptor material. The rate of FRET is dependent on distance between the dipoles following an inverse power law ${ }^{38}$ :

$$
\Gamma_{F R E T}=\frac{1}{\tau}\left(\frac{R_{0}}{R}\right)^{-6}
$$

Where $\tau$ is the fluorescence lifetime of the donor in seconds, $R_{0}$ is the system specific Förster radius in meters. When the inter-exciton distance $R$ is equal to or smaller than $R_{0}$ the transfer rate is equal to or higher than the fluorescence lifetime. Equation 7 is obtained for molecular interactions of Frenkel excitons ${ }^{38}$ which can be approximated by a quantum dot confinement. This relation can also be applied when the quantum dot quantum dot interactions are considered. However, recent studies have shown that exciton dimensionality, i.e. confinement to a quantum dot, wire or well, affects strongly the power coefficient in equation $7^{39,40}$. The efficiency of the energy transfer is also dependent on the relative orientation of the dipole moments, $\widehat{\boldsymbol{u}}$, of the donor and acceptor $^{41}$, where parallel alignment is preferred. 
The energy transfer from the donor material corresponds to the joule loss of the electric field created by the oscillation of the dipole polarization of the donor. The field penetrating into the acceptor medium has to be in resonance with the natural frequency of the acceptor dipole. The joule loss, $Q(\omega)$, in an isotropic material in such a case can be calculated as ${ }^{38}$ :

$$
Q(\omega)=\frac{\omega}{2 \pi} \int \varepsilon^{\prime \prime}(\vec{r}, \omega)|E(\vec{r})|^{2} d \vec{r}
$$

Where $\varepsilon^{\prime \prime}(\vec{r}, \omega)$ is the imaginary part of the isotropic dielectric tensor of the acceptor, $\omega$ is a frequency of the donor exciton which is resonant with the natural frequency of the acceptor. $E(\vec{r})$ is the penetrating electric field. It has been shown that this process is very fast, on the order of several $\mathrm{ps}^{42}$. That rate should be compared to the life time of excitons which is generally rather long in quantum wells. That means that the FRET from the quantum well exciton to the exciton in the acceptor material can occur faster than other radiative and non-radiative recombination processes. Figure 8 shows a schematic drawing of a GaN/polyfluorene based hybrid structure.

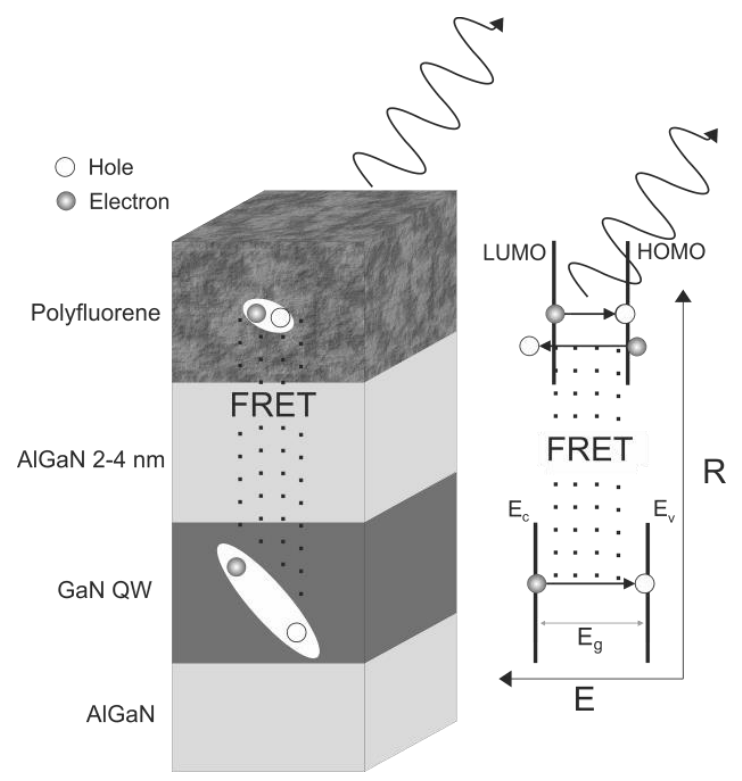

Figure 8. Illustration of the hybrid structure where FRET occurs between the Wannier-Mott exciton (i.e. energy donor) in the GaN QW and the Frenkel exciton (i.e. energy acceptor) in the polyfluorene over layer. The corresponding energy diagram is illustrated on the right side of the structure. 
The FRET occurring between the Wannier-Mott exciton in GaN quantum well and the Frenkel exciton in the polyfluorene are indicated by the dotted lines. The right part of figure 8 illustrates a corresponding energy diagram for the FRET process. The excitation of the acceptor exciton is non-radiative through energy dissipation of the electric field constructed by the Wannier-Mott exciton. Therefore, the distance between donor and acceptor dipoles (or the spacer layer) needs to be short. The influence of this distance on FRET efficient is discussed in Paper I. 


\section{Introduction to Vacuum Theory}

The demand for good quality materials keeps increasing as new technologies emerge. By fabricating thin films in a high vacuum, better control of the contamination level can be achieved. Additionally, in vacuum, the mean free path of particles increases, which allows atoms to reach the substrate easily. There are 5 vacuum regimes based on pressure regions, as summarized in Table 3 . This work uses a reactive direct current magnetron sputter system with a base pressure of $\sim 1.3 \cdot 10^{-6} \mathrm{~Pa}$.

Table 3. Definitions of different vacuum regimes and their pressure ranges in Pascal

\begin{tabular}{l|c|} 
Type of vacuum & Pressure range (Pa) \\
\hline Low vacuum & $10^{5}>\mathrm{P}>3.3 \cdot 10^{3}$ \\
Medium vacuum & $3.3 \cdot 10^{3}>\mathrm{P}>10^{-1}$ \\
High vacuum & $10^{-1}>\mathrm{P}>10^{-4}$ \\
Very high vacuum & $10^{-4}>\mathrm{P}>10^{-7}$ \\
Ultra-high vacuum & $10^{-7}>\mathrm{P} \geq 0$ \\
\hline
\end{tabular}

A central physical quantity in vacuum theory is the mean free path $(\lambda)$. The mean free path is the average distance a particle travels between collisions. An expression for mean free path could be written as ${ }^{43}$ :

$$
\lambda=\frac{k_{B} T}{\sqrt{2} \pi d^{2} p}
$$

Were $k_{B}$ is the Boltzmann's constant $\left(1.3806488 \cdot 10^{-23} \mathrm{~m}^{2} \mathrm{~kg} / \mathrm{s}^{2} \mathrm{~K}\right), T$ is temperature in Kelvin, $d$ is the diameter of the gas particle in meters and $p$ is the pressure in Pascal. A high vacuum means that the mean free path is long, i.e. the particle can travel rather far without colliding. At the base pressure of $\sim 10^{-6} \mathrm{~Pa}$ in the vacuum chamber the mean free path is around $600 \mathrm{~m}$ while at the deposition pressure $(\sim 0.67 \mathrm{~Pa})$ the mean free path is only several millimeters. 
Other central physical quantities are the Knudsen (Kn) and Reynolds (Re) numbers. Reynolds number is a way of determining flow while the Knudsen number gives information about the nature of the gas. The Knudsen and Reynolds numbers can be expressed as:

$$
\begin{gathered}
K n=\frac{\lambda}{L} \\
R e=\frac{U \rho L}{\eta}
\end{gathered}
$$

Where $L$ is the length between the chamber walls measured in meters, $U$ is the flowing stream velocity in the pipe in $\mathrm{m} / \mathrm{s}, \rho$ is the mass density $\left(\mathrm{kg} / \mathrm{m}^{3}\right)$ and $\eta$ is the viscosity $\left(\mathrm{kg} / \mathrm{m}^{3} \mathrm{~s}\right)$ of the gas. Different regions of flow can be distinguished and predicted by these dimensionless numbers. Figure 9 provides an overview of these regions. At the lowest pressure there are no gas-to-gas interactions. This region is called the molecular flow regime and is characterized by a Knudsen number bigger than 1 . Reynolds number has no physical meaning in this region. The molecular flow regime is the best known regime $^{44}$. For Knudsen numbers between 1 and 0.01 there is a transitional flow regime where the flow can be either viscous or molecular.

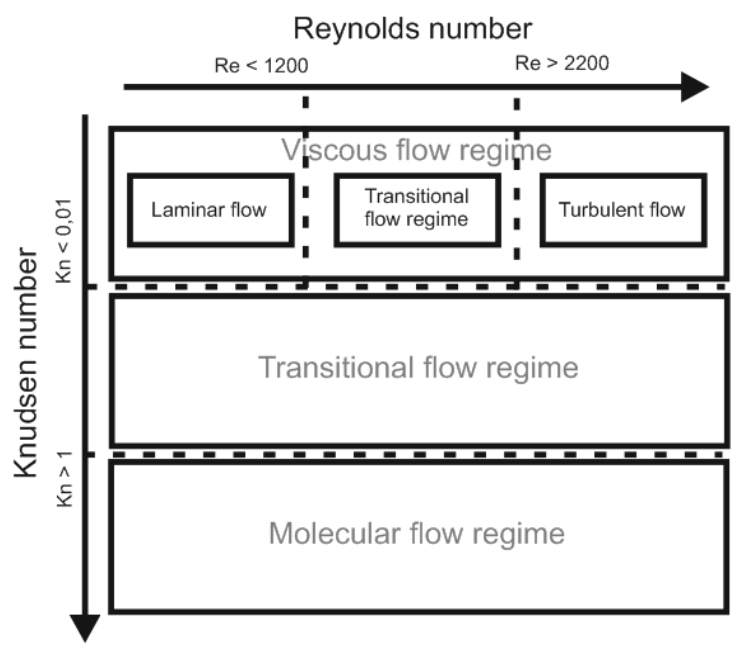

Figure 9. Schematic overview of Knudsen number vs Reynolds number for gas flow regimes. 
For Knudsen numbers less than 0.01 the gas is found in a viscous flow. The viscous behavior can be categorized into two types of flow. At Reynold numbers less than 1200 , the gas is flowing in parallel layers with no lateral mixing, and this is called a laminar flow. At Reynold numbers higher than 2200 the gas is flowing in a shifting manner and undergoes rapid variations in local pressure and velocity. This regime is known as turbulent flow. The turbulent flow is more difficult to understand and describe. At Reynolds numbers between 1200 and 2200 there is a transitional regime where the gas flow can be either laminar or turbulent. 


\section{Introduction to Sputter Deposition Theory}

Sputtering is the process in which target material is ejected by ion bombardment ${ }^{45,46}$. Sputtering can be used to clean a surface or construct microstructures. It can also be used to deposit films composed of the target material, and this is called sputter deposition. Sputter deposition is a physical vapor deposition (PVD) technique in contrary to e.g. chemical vapor deposition (CVD) techniques. Sputtering was first reported by Sir William Robert Grove ${ }^{47}$ while studying the electrical conductance of gases in the mid- $19^{\text {th }}$ century. Grove and his contemporaries, however, attributed this to thermal processes. Today, as mentioned, there is an understanding that sputtered atoms are ejected by impinging ions. The source of these ions can be plasma. The term "plasma" was first coined by Tonks and Langmuir ${ }^{48}$ in 1929. Plasma is a quasi-neutral collection of electrons, neutral particles and ions exhibiting both gaseous and liquid characteristics. Many of them glow in beautiful colors due to the de-excitation of metastable energy states of the species involved in the process. Plasmas are created by ionizing gas, e.g. by having gas entering a chamber and applying a negative potential to the target. In direct current sputter deposition, the target essentially acts as a cathode and the chamber walls act as the anode. Positive ions are accelerated towards the negative target by Coulomb's force, at the same time electrons are repelled. Ions that hit the target surface will set off a collision cascade. The transferred energy from ion to target is proportional to the mass of the target atom and the incident ion ${ }^{43}$ :

$$
E_{\text {transfer }} \propto \frac{4 M_{\text {target }} M_{\text {ion }}}{\left(M_{\text {target }}+M_{\text {ion }}\right)^{2}} \cos (\theta)^{2}
$$

Where $M_{\text {target }}$ and $M_{\text {ion }}$ are the atom masses of the target and ion in kilograms, respectively. Similar atom masses are preferable in the sputtering process. $\theta$ is the angle between the direction of the incident ion before the collision and the direction of the target atom after the collision as illustrated in figure 10. If the transferred energy in the collision cascade is high enough to overcome the surface binding energy a target atom can be ejected (sputtered). 
The rate at which this occurs is called sputter rate. From equation 12, it shows that compound targets (targets containing more than one element) will have different sputtering rates for each species in the compound. During sputtering reflected neutrals and secondary electrons are also emitted and collision cascades can occur all illustrated in figure 10 .

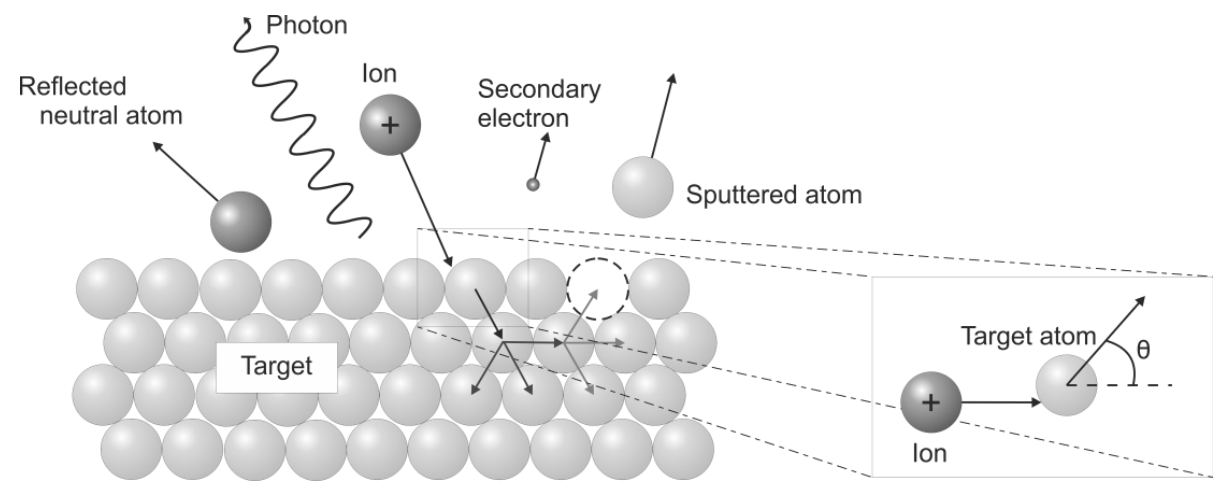

Figure 10. Schematic illustration of the sputter process. The most important processes are; the formation of sputtered atoms and collision cascades. The formation of photons and secondary electrons and reflected neutral atoms formed from ion interaction with surface are also included. The drawing to the right illustrates the definition of angle $\boldsymbol{\theta}$ in equation 12 .

The sputter deposition is a line of sight technique, which makes it hard to deposit into deep trenches. However, the sputter deposition can be done at much lower temperatures than CVD, which is highly appreciated in electronic industry where the substrates can be temperature sensitive such as organics. If sputtering is done in very high vacuum, which is the case in our laboratory, it is also a very clean method (in respect to impurity concentration).

\subsection{Reactive Sputtering}

Sputter deposition could be used to deposit e.g. metal films with low concentration of impurities. That would, however, require that the gas used in the sputter process do not form chemical bonds to the metal at the substrate. The way to assure this is to use inert gas, such as noble gases. Argon is relatively cheap and is often used as a working gas. 
On the other hand, sputtering can have complicated reactive processes, and is called reactive sputter deposition if there is a chemical reaction forming the film. For instance, if a reactive gas, such as oxygen or nitrogen is used to react with sputtered material, compounds, oxides or nitrides can be formed by chemical reactions at the substrate surface $^{49,50}$. A rather common approach is to use a mixture of inert and reactive gases. In this work, gallium nitride was fabricated by the reaction of sputtered gallium atoms from the target in a pure nitrogen gas atmosphere. When sputtering in a pure reactive atmosphere, the target can get poisoned. Poisoning is when the reactive gas inside the plasma starts to react with the target surface. The target needs to be negatively charged to attract positive ions and if the target is poisoned due to the formation of an insulating film, an observed phenomenon called arcing occurs. Arcing is disadvantageous in DC sputter deposition since it donates large amounts of energy to the target surface which can eject macro-particles that destroy the film. Even if the poisoning forms conductive films, it is still worth avoiding because the process will be different from sputtering using a new target. There will be a change in surface binding energy, introducing a change in sputtering rate.

\subsection{Magnetrons}

In a basic sputter process, secondary electrons can be ejected. In such cases they are repelled by the negative cathode and lost to the chamber walls. A magnetron can be used to spatially contain the electrons and maintain the plasma close to the target surface. Magnetrons are constructed to produce a magnetic field over the target in such a way that the field is, parallel to, and in the vicinity of, the target surface. Secondary electrons produced during the sputter process are trapped in this magnetic field due to Lorentz forces, $\vec{F}$ :

$$
\vec{F}=q(\vec{E}+\vec{v} \times \vec{B})
$$

Where $q$ is the particle charge (in this case the elementary charge in Coulomb), $\vec{E}$ is the electric field in Joule, $\vec{v}$ is the particle velocity in $\mathrm{m} / \mathrm{s}$, and $\vec{B}$ is the magnetic field in N/Am. The trapped electrons continue to ionize neutral atoms close to the target, and thereby sustain the plasma. The higher ionization rate obtained using a magnetron increases the sputter rate and in turn the deposition rate in general ${ }^{43}$. Because sputtered atoms are neutral, they are unaffected by the magnetic field.

Fundamental plasma physics implies that the pressure must be sufficient for the particle density to sustain the plasma. The second advantage of using a magnetron is that 
the pressure can be decreased further while still maintaining the plasma thanks to the higher ionization rate. By lowering the pressure in the chamber, films can be produced with a higher purity.

Figure 11 illustrates a typical magnetron consisting of two strong concentric circular magnets placed behind the target in one of three configurations. Two types of arrangements of magnet poles directed towards the target are commercially available. That is, North-South-North and South-North-South arrangement. In an unbalanced type I magnetron there is a stronger core magnet than the compensating outer surrounding magnet. In the balanced magnetron these two magnets are balanced in strength. In the unbalanced configuration of type II we have the opposite situation to type I. The surrounding magnet is stronger and cannot be balanced by the core magnet. The unbalanced type II magnet is preferable for deposition techniques since the higher plasma densities can be achieved close to the substrate without increasing substrate bias. The increasing of substrate bias is accompanied by formation of defects and also by incorporated stresses ${ }^{51,52}$. The higher plasma densities close to the substrate activate the sputter gas and also increase the ion bombardment which is used to modify the properties of the growing film.

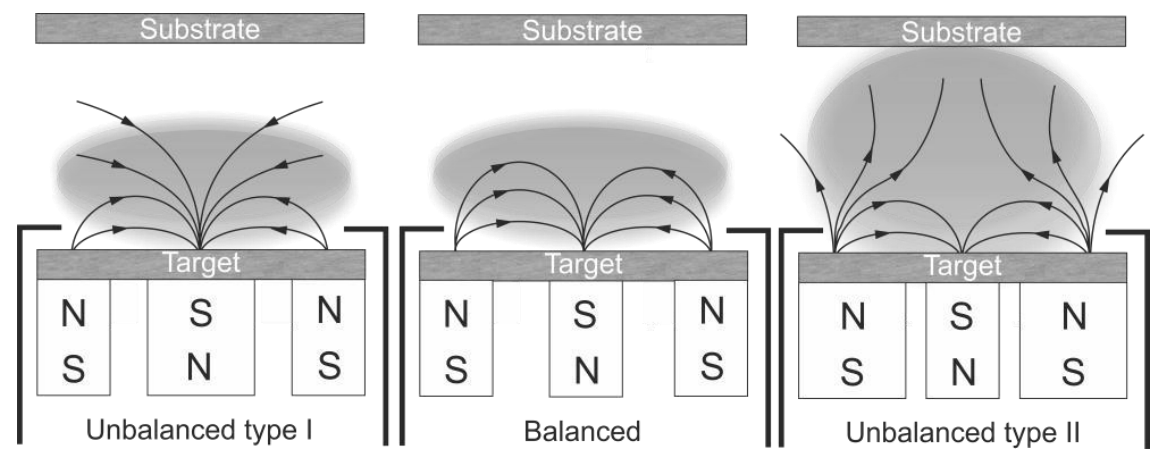

Figure 11. Schematic illustration of three types of magnetrons, all are of North-South-North configuration and of either unbalanced type I, II or the balanced type. Unbalanced type II magnetron is the preferred configuration for deposition systems.

A disadvantage of the magnetron is that the target erosion will occur in the position where the magnetic field is perpendicular to the target surface. This will be seen as a dimple in the target over the middle magnet as well as a trench (also called "racetrack") in the target over the surrounding magnet. This effect results in a reduction of target material that can be used during the lifetime of the target. 


\subsection{The Reactor}

The chamber used for the growth of GaN NRs is called Nidhögg. The base and working pressure are measured with an ion gauge and a capacitance manometer, respectively. The base pressure is in the range of $10^{-8}$ Torr $\left(1.3 \cdot 10^{-6} \mathrm{~Pa}\right)$. The reactor is constructed in stainless steel with copper gaskets for sealing on all flanges except on the Ga magnetron. A schematic illustration is shown in figure 12. The chamber is connected to a transfer tube and a load lock chamber, thus the growth chamber is not exposed to the atmosphere during the sample loading. The pumping speed can be regulated with a butterfly baffle valve. The process gas flow rates are controlled with mass flow controllers calibrated for the specific gases. The purity of $\mathrm{Ar}$ and $\mathrm{N}_{2}$ gas can be reached to $99.999999 \%$ by using gas purifiers. A $99.99999 \%$ pure Ga target is contained in a water-cooled horizontal $50 \mathrm{~mm}$ stainless steel trough mounted on a type II unbalanced magnetron. Ga has a melting temperature of $29^{\circ} \mathrm{C}$ and will therefore be liquid during the deposition. The gallium target being a liquid provides two advantages:

1. No target erosion

2. Continous supply of fresh material to the surface.

Ga target is prepared from melting pellets directly into the steel trough. The target is solidified by placing the trough on a liquid nitrogen cooled holder. After being installed in the chamber again, the target is thouroghly clean-sputtered in pure Ar gas before usage.

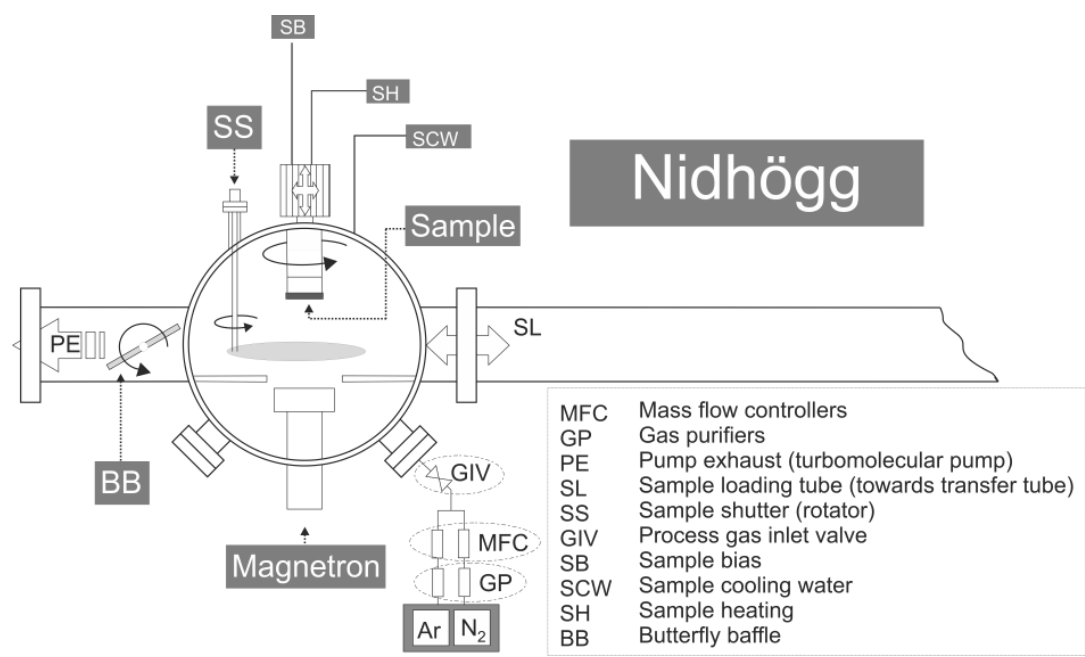

Figure 12. Illustration of the reaction chamber Nidhögg (side view). 


\section{Metal-Organic Chemical Vapor Deposition}

Metal-organic chemical vapor deposition (MOCVD), is a method of chemical synthesis of thin films using metal-organic gaseous reagents. These reagents are called precursors. CVD is not a line of sight technique compared to, for example, sputter deposition. CVD can therefore be used to coat much more complex surfaces while achieving a more uniform thickness.

CVD have a long tradition within the fabrication of photonic and electronic device structure, and great control of the deposition is possible ${ }^{43,53}$. CVD process can be considered as a series of steps, illustrated in figure 13. First there is a transport of precursors into the reactor, then the following processes can be mentioned: diffusion or convection of precursors through the boundary layer, possible gas phase reaction, adsorption of the precursors, surface diffusion, surface chemical reaction, desorption of adsorbed precursors, desorption of reaction by-products and mass transport of byproducts out of the chamber.

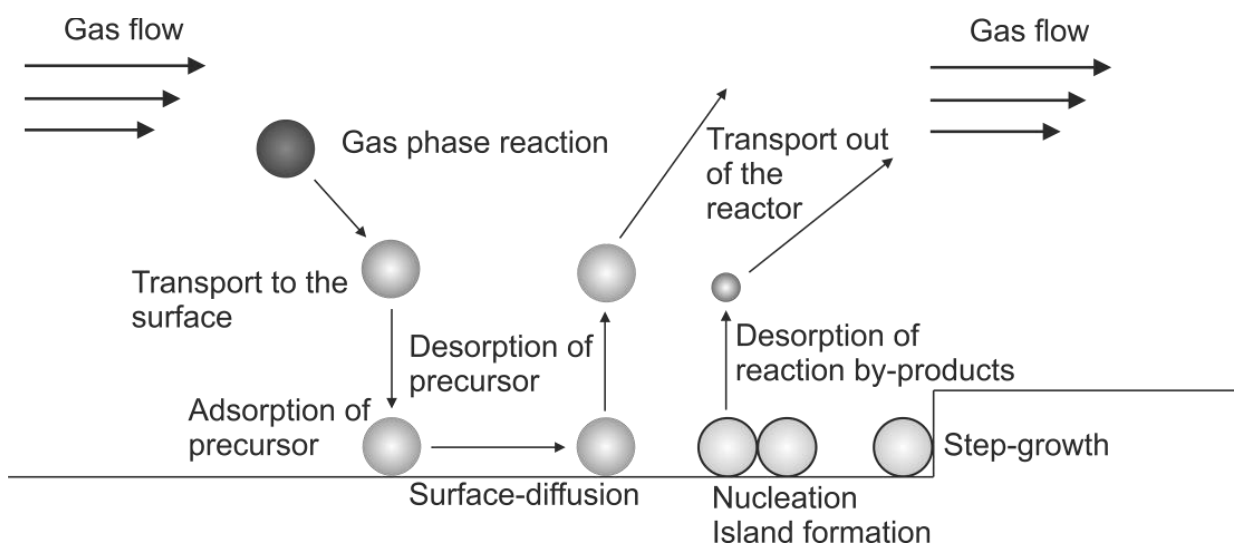

Figure 13. Illustration of the CVD process on precursor level near substrate surface. 
A "good" precursor must possess as many as possible of the following properties:

- High volatility

- High purity

- Be non-toxic

- Be available at low cost with consistent purity

- Have good thermal stability during evaporation and transport into the reactor

- Clean decomposition

- Give non-hazardous by-products

Usually, not all of these requirements can be met. Volatility is one of the top priorities. Therefore, ligands that form hydrogen bonds, or strong dipole-dipole or Van-der-Waals interactions should be reconsidered ${ }^{54}$.

There are two main choices when it comes to types of precursors; single source precursors or combined multiple precursors. The single source precursor contains all the elements desirable in the film in the form of one single molecule. This requires a higher planning of chemistry to make sure that the composition is also reflected in the precursor. The different bonds within the molecule must reflect the desired film. Some bonds are supposed to be in the film and have to be stronger, while some weaker bonds are supposed to break to release the supporting backbone of the precursor. Besides being more chemically hard to produce they also generally suffer from high molecular weight which gives them less volatility. However, when done correctly, the single source precursor has a clear advantage, because of a much better reproducibility can be achieved compared to the case of multiple sources where fluctuations in flow can cause fluctuations in stoichiometry ${ }^{53}$.

For group III-N growth with MOCVD, the following typical main precursors are usually used: trimethyl-gallium, trimethyl-aluminum and trimethyl-indium. Ammonia is used as the source of nitrogen and hydrogen or nitrogen can be used as carrier gas. Metal organic precursors are stored in bubblers connected to the gas system of the growth reactor. The process of decomposition inside a growth chamber can be complicated and include several steps, for example: trimethyl-gallium decomposes first to dimethyl-gallium and then monomethyl-gallium ${ }^{55,56}$. 


\subsection{Hot Wall vs Cold Wall}

A CVD reactor can be designed into two main types; hot wall or cold wall. In conventional CVD, heat is the driving force which initiates the reactions. In cold wall reactors the substrate has higher temperature than the surrounding wall. This will decrease the reaction rate on the chamber walls and increase the precursor efficiency and consequently also the deposition rate on the substrate compared to the hot wall reactors. In hot wall reactors the whole reactor chamber is heated. In this case, a more uniform temperature distribution across the substrate can be achieved. Temperature gradients and fluctuations across the substrate are undesirable since it cause fluctuations in reaction rates, diffusion rates, and are detrimental to overall control of the film. However, in hot wall reactors, the gas phase reactions occur over the entire chamber which makes the control of the precursors partial pressure more difficult ${ }^{53}$.

\subsection{Growth Rate}

Growth rate, $k$, depend on temperature, $T$ in Kelvin, of the substrate and activation energy, $E_{A}$ in Joule, of the reaction according to the Arrhenius equation:

$$
k=A e^{-\left(E_{A} / R T\right)}
$$

Where $R$ is the gas constant $(8.3144621 \mathrm{~J} / \mathrm{Kmol}), A$ is the pre-exponential factor (in reciprocal seconds for first order reactions) and is representing how often molecules collide in the proper orientation. Three regimes of growth rate can be distinguished depending on temperature. These regimes are illustrated in figure 14. At low temperatures we find a so called kinetically limited regime. The low temperature means that the available energies are too small for most precursors to overcome the activation energy of the surface reactions. In the intermediate temperature regime, the temperatures are sufficient for precursors to overcome the energy barrier of reaction once the species reach the surface. Reaching the surface, which is done by diffusion, is the limiting step. This temperature regime is considered preferable because it is the most stable regime in respect to temperature fluctuations. Also, the growth rate in this regime can be conveniently changed by adjusting the partial pressure of the precursors, which is called a feed-rate-limited process. 


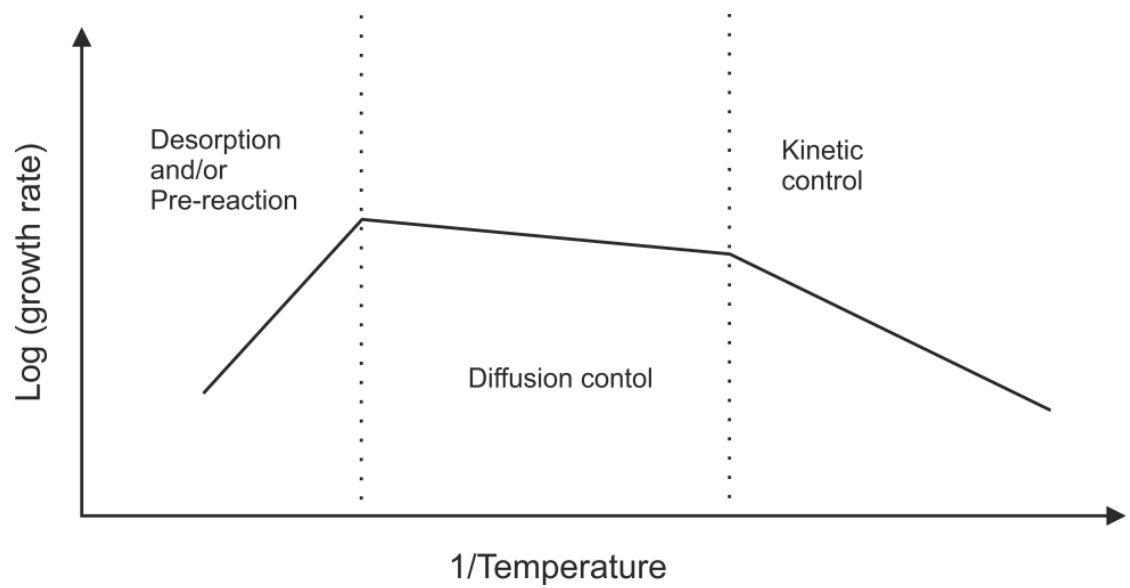

Figure 14. Illustration of how the growth rate (in a logarithmic scale) changes with reciprocal temperature according to Arrhenius equation. Specific temperatures for each region are highly dependent on activation energy of the precursor.

At high temperatures all of the precursors decompose and react but the temperature is so high that the thermodynamics can change. A gas phase reaction leading to a solid film can be described as:

$$
X(g)+Y(g) \leftrightarrow X Y(s)
$$

Here, the arrow pointing to the right means solid film formation and vice versa. The change in the Gibbs energy, $\Delta G$ in $\mathrm{J} / \mathrm{mol}$, can be seen as the "driving force" for the direction in which the reaction will proceed spontaneous

$$
\Delta G=G_{X Y}^{S}-\left(G_{X}^{g}+G_{Y}^{g}\right)
$$

And where the sign of $\Delta G$ can be translated according to

$$
\Delta G=\left\{\begin{array}{rr}
>0 & \text { Parasitic reactions }=\text { reaction (15) going left } \\
=0 & \text { Equilibrium } \\
<0 & \text { Film formation }=\text { reaction (15) going right }
\end{array}\right.
$$

At very high temperatures, parasitic reactions become favorable, and the growth rate decreases. Also, in this regime the reaction rates increase in other parts of the chamber which results in the diluted precursor concentrations. 


\section{Characterization}

\subsection{X-ray Diffraction}

X-ray diffraction (XRD) was discovered first by Laue et. al. ${ }^{57}$ in 1912. It is a technique used for investigating the crystal structure of the material. For diffraction to occur, electromagnetic radiation has to impinge on a periodic structure that has a periodicity in the same order of length as the wavelength of the incoming wave. This is true for all periodic structures and all propagating waves. For example, diffraction could even be produced by letting a red laser pen shine through a feather of a bird.

Atoms arranged in a crystal, however, are arranged with an interatomic length scale of a few nanometers. The wavelength of visible light is then too long, and instead, $\mathrm{X}$-rays are used. As X-rays impinge on a material surface, three processes can occur; photoionization, Compton scattering or Thomson scattering. In Thomson scattering the energy from the electromagnetic wave is elastically scattered by the electrons. Therefore the wavelength of the X-ray is preserved. It's this scattering process that is used in XRD. Figure 15 illustrates this scattering process. As the wave fronts scatter against the crystal new wave fronts are created. These will then interfere with each other, either destructively or constructively.

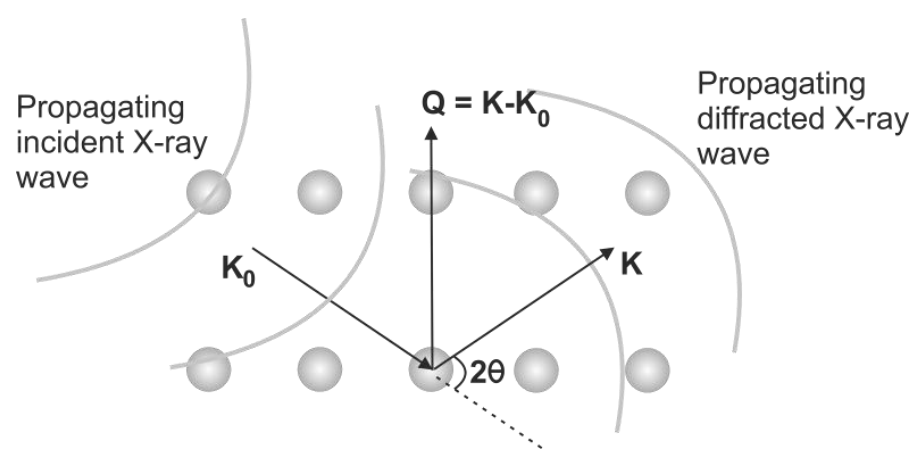

Figure 15. Illustration of X-ray interaction with a crystal structure.

Note that this is not a reflection of X-rays but diffraction. In figure 15 the so called scattering vector, (or reciprocal space vector) $Q$ in reciprocal meters, is also defined as: 


$$
Q=K-K_{0}
$$

Where $K_{0}$ and $K$ are the incident and diffracted wave vectors of the electromagnetic field with wavelength, $\lambda$ in meters. If elastic scatter then:

$$
\left|K_{0}\right|=|K|=\frac{2 \pi}{\lambda}
$$

Constructive interference occurs when ${ }^{58}$ :

$$
\left\{\begin{array}{l}
|Q|=2\left|K_{0}\right| \sin \theta=\frac{4 \pi}{\lambda} \sin \theta \\
|Q|=\frac{2 \pi}{d_{h, k, l}}
\end{array}\right.
$$

Where $d_{h, k, l}$ is the interplanar spacing in meters. From this we will obtain the Braggs law

$$
\lambda=2 d_{h, k, l} \cdot \sin \theta
$$

Since $\lambda$ of a specific source is known and $\theta$ can be detected, thus, $d_{h, k, l}$ can be obtained. However, also other considerations have to be done in order to model and understand a diffractogram, e.g. a geometrical structure factor and an atomic form factor. In this work $\theta-2 \theta$ scans were performed in a Phillips 1820 Bragg-Brentano diffractometer. The measurement geometry of this experimental setup is illustrated in figure 16.

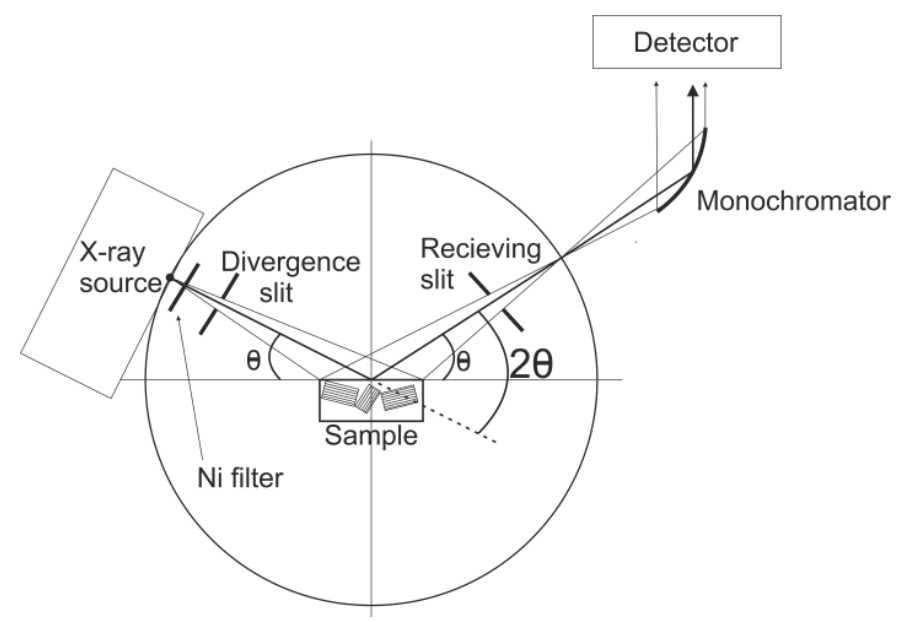

Figure 16. Schematic illustration of the Bragg-Brentano geometry used in Paper II. 
In a $\theta-2 \theta$ scan the incident beam angle and the detection angle is simultaneously changed. Expected diffractogram peaks for powder samples are found in reference data bases. Samples like thin films can exhibit specific preferred crystal alignment in relation to the incident beam and will therefore display peaks with a different intensity ratios than a powder sample.

When performing a scan in XRD, it is important to keep track of the scatter vector in reciprocal space, see figure 17. XRD is a technique that maps the reciprocal lattice points of the crystal structure. By performing a $\theta-2 \theta$ scan, the scan direction shown in figure $17 \mathrm{a}$ is perpendicular to the surface. We only scan along the direction of the scattering vector. Also, a rocking curve scan ( $\omega$-scan) is illustrated in figure 17b. A quantitative estimation of the crystal quality can be done by determining the full width at half maximum (FWHM) of the obtained peak in $\omega$-scan.
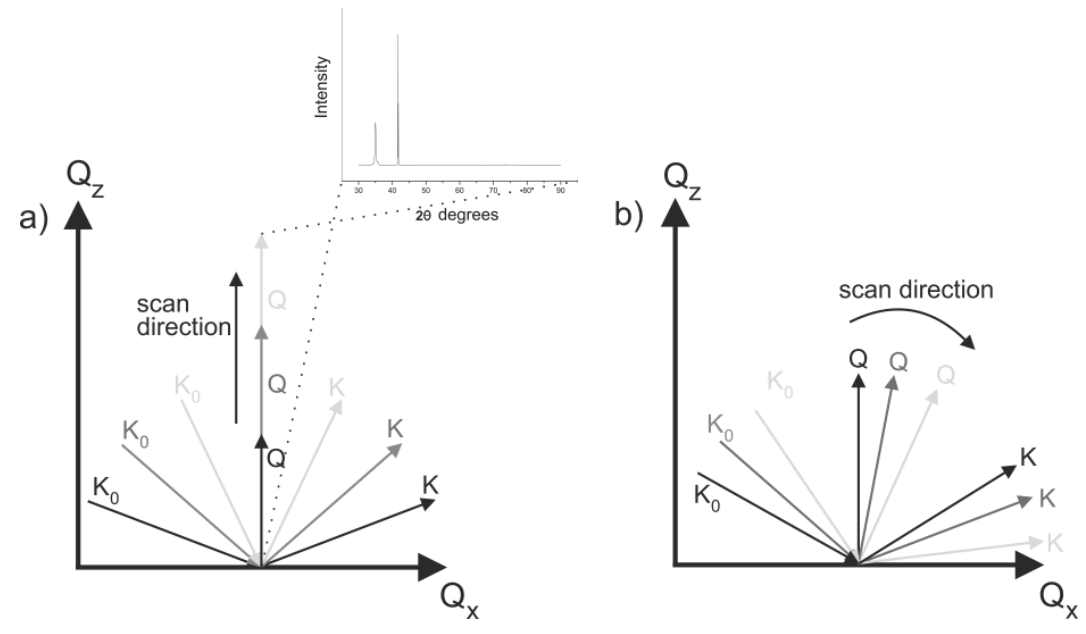

Figure 17. Illustration of scan direction in a) a $\theta-2 \theta$ scan and b) a rocking curve scan

From the reciprocal space map a lot of information such as the in-plane lattice parameter and film strain can be obtained. XRD is therefore a very powerful nondestructive technique to study structural crystal quality. No time consuming preparation of samples is needed for the investigation of thin films by XRD. 


\subsection{Scanning Electron Microscopy}

Scanning electron microscopy (SEM) is a technique used to image much smaller features than what is possible in visible-light optical microscopy. SEM is a widely used tool to examine microstructures and morphology. Visible-light optical microscopy operates with visible wavelengths in the range of 400-700 nm. An electron microscope such as SEM uses an electron beam instead. The resolution that can be obtained is dependent on wavelength, $\lambda$. Typical electron beam energies are $0.5-40 \mathrm{keV}$. The wavelength of an electron beam can be calculated as ${ }^{59}$ :

$$
\lambda=\frac{h}{\sqrt{(2 m E)}}
$$

Where, $h$, is Plank's constant $\left(6.62606957 \cdot 10^{-34} \mathrm{Js}\right), E$ is the kinetic energy in Joule, and $m$ is the electron mass in kilograms. Thus, the wavelength of the electron becomes much smaller than for visible light ( $1 \mathrm{~nm}$ vs. $400-700 \mathrm{~nm}$ ).

In SEM, the electron beam is probing the sample surface. When examining the SEM images it is important to take into account the electron beam energy. With increasing energy the so called excitation volume illustrated in figure 18 is increased. When an electron beam impinges on a material a lot of processes occur that produces different kinds of radiation, such as; X-rays, Auger electrons and cathodoluminescence. Also figure 18 illustrates which depth corresponding to signal for different process.

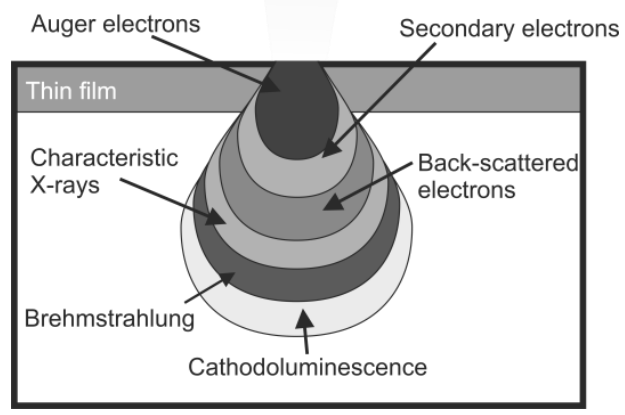

Figure 18. Schematic drawing of excitation volume and the regions from where each type of signal can be measured. 
An impinging electron beam will create an electron surplus across the sample. The sample becomes electrically charged. A negatively charged sample will start deflecting the electron beam. When the beam is deflected in this manner the image is drifting. Preferably the sample should be conductive and grounded to mitigate this effect. However, if the sample is a poor conductor or insulator, lowering the beam energy, beam current and grounding of the sample surface can be used to improve the quality of the imaging. Samples can also be coated by a thin metal film (usually gold or platinum) before SEM study. The problem with coating is that the topology can be slightly altered.

An SEM is composed of an electron gun, condenser lenses, deflection coils, an objective lens, sample holder and detectors placed within a vacuum chamber. Our system is a LEO 1550 Gemini SEM with a Field emission gun (FEG) as an electron source. One of the most important advantages with FEG is the superior brightness compared to a thermionic gun even at much lower acceleration voltages. Brightness is the current density per unit solid angle of the source. A high brightness means more electrons can be aimed at the sample so that more information can be obtained while maintaining the spot size of the beam ${ }^{59}$. A FEG creates electrons by applying several $\mathrm{kV}$ to a very thin tip to have it emit electrons. The LEO 1550 system has a so called Schottky FEG electron gun which means a heating current is also applied to enhance the emission. When electrons leave the gun they enter a set of lenses. In all electron microscopes the lenses are carefully engineered magnets. When the electrons pass through a magnetic field it will experience a Lorentz force. As a result, the beam can be controlled and focused. In a basic SEM the electrons that leave the electron gun will go through one or two condenser lenses in order to be focused. After this they pass through the deflection coils. The deflection coils create a magnetic field in order to steer the electron beam so that it scans the surface. The objective lens is the lens situated between the deflection coils and the sample. This lens is responsible for focusing on the surface so that the impinging beam radius is small. In SEM the secondary electrons or the back scattered electrons are detected to form an image. Since secondary electrons are electrons emitted from the outer shell of the material atoms they have low energies, so they will come from confined places close to the surface. This means that detecting secondary electrons will give a good topographical contrast. Back scattered electrons are more scattered the heavier the nuclei and do therefore give a compositional contrast. However, SEM images do not give elemental information of the sample. 


\subsection{Transmission Electron Microscopy}

Transmission electron microscopy (TEM) was first developed in 1932 by Knoll and Ruska ${ }^{60}$. TEM is also an electron microscope similar to SEM. However, there are some significant differences. SEM is a technique that produces images from detecting secondary and back scattered electrons while TEM produce images from detecting transmitted electrons. Electrons need to be transmitted in order to reach the fluorescent screen or CCD below the sample. Therefore, TEM requires a very thin sample transparent to electrons. This mean, that TEM is a destructive technique since the sample needs to be cut and polished to the proper thickness. Another difference is that TEM operates at much higher beam energies (100-300 keV) which allows for higher spatial resolution (Equation 21 still applies). In this thesis, samples were studied with a high resolution FEI Tecnai G2 200 keV FEG scanning electron microscope. Since TEM uses the information of transmitted electrons, both images and electron diffraction patterns similar to X-ray diffraction patterns can be produced. Being able to combine the high spatial resolution with information about the crystal structure makes TEM a very powerful characterization technique.

\subsection{Cathodoluminescence}

As discussed in Chapter 3 when an electron beam impinges on a material surface, electrons of the target material can be excited and then radiatively recombine with a hole. The process is called cathodoluminescence (CL). The CL detectors can be integrated with either an SEM or a TEM. CL is a useful technique to investigate emission from semiconductors and also from insulators with large band gap because the energy of the electron beam is higher than for most of the lasers. Our lab has a Gatan MonoCL4 cathodoluminescence system used combined with an SEM. It employs a so called Czerny -Turner type monochromator, the principal parts of which are two slits, two spherical mirrors and a diffraction grating. Another big advantage with measuring luminescence using an electron beam as excitation source is the high spatial resolution of the same order as in the SEM or the TEM. Thus, one can determine from which region of the sample the luminescence originates. SEM and CL can be detected simultaneously allowing direct comparison of SEM and CL images. Different defects, dopants and crystal structures emit light of different photon energy which enables identification of these centers by obtaining a luminescence spectrum. Because of the relatively large excitation volume of the CL plume shown in figure 18, depth-resolved information can be obtained. 
CL measurements can be conducted at low temperatures. The sample is cooled down by liquid helium. Our CL instrument has the possibility of attaching a liquid $\mathrm{He}-$ cooled cryo-stage for sample mounting. Low temperature measurements enhance radiative recombination because non-radiative recombination centers activate with increasing temperature. Also, thermal broadening of the exciton energy is avoided ${ }^{26,61}$.

Charging is a problem for CL measurements in the same way as for SEM. In contrast to SEM, the sample cannot be metal coated because that prevents the luminescence signal.

\subsection{Photoluminescence and Time-Resolved Photoluminescence}

When an electron is excited by a laser or a lamp (i.e. by photons) and recombines emitting photons, the process is called photoluminescence (PL). PL has some advantages over CL. For example a laser of lower energy than the width of the band gap can be chosen to probe for defects, donors or acceptors inside the band gap ${ }^{62}$. In this work, we have used time-resolved photoluminescence (TRPL) which gives information about the PL recombination dynamics. For TRPL measurements a Ti:sapphire femtosecond solid state laser was used. The pulse frequency was $75 \mathrm{MHz}$. We have used, for PL excitation, the third harmonics with the wavelength $266 \mathrm{~nm}$ which corresponds to the energy of $4.65 \mathrm{eV}$ (i.e. above $\mathrm{GaN}$ band gap). In order to study TRPL at low temperatures, the samples were cooled in a cryostat. Short laser pulses illuminate the sample surface and the emission from the sample is collected and focused by lenses into a monochromator. The signal is detected by a streak camera. The streak camera is transforming a temporal profile of light into a spatial profile on a CCD. The light entering the streak camera produces electrons when impinging on a photocathode surface. The electrons are accelerated through a pair of electrodes that will slightly deflect them with an applied voltage that varies smoothly with time. After this, the electrons will impinge on a thin phosphor screen that will emit light. The light is collected by the CCD behind the screen. The result is an image where energy is increasing in positive horizontal direction and time increase in negative vertical direction. The number of impinging photons per CCD pixel determines the intensity. 


\section{Bibliography}

1. Mishra, U. K. \& Kazior, T. E. GaN-Based RF Power Devices and Amplifiers. Proc. IEEE 96, 287 (2008).

2. Morkoç, H. et al. Large-band-gap SiC, III-V nitride, and II-VI ZnSe-based semiconductor device technologies. J. Appl. Phys. 76, 1363 (1994).

3. Pearton, S. J., Zolper, J. C., Shul, R. J. \& Ren, F. GaN: Processing, defects, and devices. J. Appl. Phys. 86, 1 (1999).

4. IUPAC periodic table of elements. (2013). at <http://www.iupac.org/fileadmin/user_upload/news/IUPAC_Periodic_Table1May13.pdf> (Last accessed Dec 2014)

5. Utsumi, W. et al. Congruent melting of gallium nitride at $6 \mathrm{GPa}$ and its application to single-crystal growth. Nat. Mater. 2, 735 (2003).

6. Manasevit, H. M., Erdmann, F. M. \& Simpson, W. I. The Use of Metalorganics in the Preparation of Semiconductor Materials. J. Electrochem. Soc. 118, 1864 (1971).

7. Maruska, H. P. Violet luminescence of Mg-doped GaN. Appl. Phys. Lett. 22, 303 (1973).

8. M. Junaid, Thesis, "Magnetron Sputter Epitaxy of GaN Epilayers and Nanorods", Linköping Studies in Science and Technology, Dissertation No. 1482, ISBN:978-917519-782-1 (2012)

9. Li, B., Zhuang, H., Xue, C. \& Zhang, S. Growth and characterization of pineneedle-shaped $\mathrm{GaN}$ nanorods by sputtering and ammoniating process. Superlattices Microstruct. 43, 262 (2008). 
10. Dong, Z. et al. Synthesis of three kinds of GaN nanowires through Ga2O3 films' reaction with ammonia. Phys. E Low-dimensional Syst. Nanostructures 27, 32 (2005).

11. Amano, H., Sawaki, N., Akasaki, I. \& Toyoda, Y. Metalorganic vapor phase epitaxial growth of a high quality GaN film using an AlN buffer layer. Appl. Phys. Lett. 48, 353 (1986).

12. Iwaya, M. et al. Reduction of Etch Pit Density in Organometallic Vapor Phase Epitaxy-Grown GaN on Sapphire by Insertion of a Low-Temperature-Deposited Buffer Layer between High- Reduction of Etch Pit Density in Organometallic Vapor Phase Epitaxy-Grown GaN on Sapphire by I. Jpn. J. Appl. Phys. 37, L316 (1998).

13. Narayanan, V., Lorenz, K., Kim, W. \& Mahajan, S. Origins of threading dislocations in GaN epitaxial layers grown on sapphire by metalorganic chemical vapor deposition. Appl. Phys. Lett. 78, 1544 (2001).

14. Dai, Q. et al. Internal quantum efficiency and nonradiative recombination coefficient of GaInN/GaN multiple quantum wells with different dislocation densities. Appl. Phys. Lett. 94, 111109 (2009).

15. Zubia, D. \& Hersee, S. D. Nanoheteroepitaxy: The Application of nanostructuring and substrate compliance to the heteroepitaxy of mismatched semiconductor materials. J. Appl. Phys. 85, 6492 (1999).

16. Bergbauer, W. et al. Continuous-flux MOVPE growth of position-controlled Nface $\mathrm{GaN}$ nanorods and embedded InGaN quantum wells. Nanotechnology 21, 305201 (2010).

17. Xiang, H.-F. et al. Organic, polymer and organic/inorganic hybrid light-emitting devices based on phosphorescent fluorinated platinum(II) porphyrin. 5519, 218 (2004).

18. Steckl, A. J., Heikenfeld, J., Allen, S. C. \& Member, S. Light Wave Coupled Flat Panel Displays and Solid-State Lighting Using Hybrid Inorganic / Organic Materials. J. Disp. Technol. 1, 157 (2005). 
19. Hide, F., Kozodoy, P., DenBaars, S. P. \& Heeger, A. J. White light from InGaN/conjugated polymer hybrid light-emitting diodes. Appl. Phys. Lett. 70, 2664 (1997).

20. Burn, P. L. et al. Chemical tuning of electroluminescent copolymers to improve emission efficiencies and allow patterning. Nature 356, 47 (1992).

21. Zhang, Y. \& Blom, P. W. M. Electron and hole transport in poly(fluorenebenzothiadiazole). Appl. Phys. Lett. 98, 143504 (2011).

22. Chin, V. W. L., Tansley, T. L. \& Osotchan, T. Electron mobilities in gallium, indium, and aluminum nitrides. J. Appl. Phys. 75, 7365 (1994).

23. Förster, T. Energiewanderung und Fluoreszenz. Die naturwisseshaften 6, 166 (1946).

24. Förster, T. Zwischenmolekulare Energiewanderung und Fluoreszenz. Ann. Phys. 6, 55 (1939).

25. Förster, T. Experimentelle und theoretische Untersuchung des zwischenmolekularen Übergangs von Elektronenanregungsenergie. Zeitschrift für Naturforsch. 4a, 321 (1949).

26. Grundmann, M. The physics of semiconductors. (Springer-Verlag, 2010).

27. Strite, S. GaN, AlN, and InN: A review. J. Vac. Sci. Technol. B. 10, 1237 (1992).

28. Müller, U. Inorganic structural chemistry. (Wiley-VCH Verlag Gmbh \& Co. KGaA, Weinheim, 2007).

29. Waltereit, P. et al. Nitride semiconductors free of electrostatic fields for efficient white light-emitting diodes. Nature 406, 865 (2000).

30. Vurgaftman, I. \& Meyer, J. R. Band parameters for nitrogen-containing semiconductors. J. Appl. Phys. 94, 3675 (2003). 
31. Vurgaftman, I., Meyer, J. R. \& Ram-Mohan, L. R. Band parameters for III-V compound semiconductors and their alloys. J. Appl. Phys. 89, 5815 (2001).

32. Ponce, F. A. \& Bour, D. P. Nitride-based semiconductors for blue and green light emitting devices. Nature 386, 351 (1997).

33. Albert-Seifried, S. et al. Pressure-Induced Delocalization of Photoexcited States in a Semiconducting Polymer. Phys. Rev. Lett. 105, 195501 (2010).

34. Davydov, S. Y. Estimates of the spontaneous polarization and permittivities of AlN, GaN, InN, and SiC crystals. Phys. Solid State 51, 1231 (2009).

35. Blumstengel, S., Sadofev, S., Xu, C., Puls, J. \& Henneberger, F. Converting Wannier into Frenkel Excitons in an Inorganic/Organic Hybrid Semiconductor Nanostructure. Phys. Rev. Lett. 97, 237401 (2006).

36. Schulz, S. \& O'Reilly, E. P. Excitonic binding energies in non-polar GaN quantum wells. Phys. status solidi 7, 1900 (2010).

37. Basko, D., Rocca, G. C. La, Bassani, F. \& Agranovich, V. M. Förster energy transfer from a semiconductor quantum well to an organic material overlayer. Eur. Phys. J. B 362, 353 (1999).

38. Agranovich, V. M., Gartstein, Y. N. \& Litinskaya, M. Hybrid Resonant Organic-Inorganic Nanostructures for Optoelectronic Applications. Chem. Rev. 111, 5179 (2011).

39. Rindermann, J. J. Monemar, B., Pozina, G., Hultman, L., Amano, H., \& Lagoudakis, P. G. Dependence of Resonance Energy Transfer on Exciton Dimensionality. Phys. Rev. Lett. 107, 236805 (2011).

40. Singh, H. \& Bagchi, B. Non-Förster distance and orientation dependence of energy transfer and applications of fluorescence resonance energy transfer to polymers and nanoparticles : How accurate is the spectroscopic ruler with $1 / \mathrm{R}^{6}$ rule? Curr. Sci. 89, 1710 (2005). 
41. Loura, L. M. S. Simple estimation of Förster Resonance Energy Transfer (FRET) orientation factor distribution in membranes. Int. J. Mol. Sci. 13, 15252 (2012).

42. Agranovich, V. M., La Rocca, G. C. \& Bassani, F. Efficient electronic energy transfer from a semiconductor quantum well to an organic material. J. Exp. Theor. Phys. Lett. 66, 748 (1997).

43. Ohring, M. Materials science of thin films: Deposition \& structure. (Elsevier (Singapore) Pte Ltd., 2006).

44. O'Hanlon, J. F. A User's Guide to Vacuum Technology. (John Wiley \& Sons, Inc., 2003).

45. Jakas, M. M. Transport theories of sputtering. Philos. Trans. A. Math. Phys. Eng. Sci. 362, 139 (2004).

46. Oechsner, H. Sputtering - A review of some recent experimental and theoretical aspects. Appl. Phys. 8, 185 (1975).

47. Mattox, D. M. Society of vacuum coaters. (2003). at $<$ http://www.svc.org/HistoryofVacuumCoating/History-of-VacuumCoating.cfm> (Last accessed Dec 2014)

48. Tonks, L. \& Langmuir, I. Oscillations in ionizfd gases. Phys. Rev. 33, 195 (1929).

49. Depla, D. Magnetrons, reactive gases and sputtering. (2013).

50. Berg, S. \& Nyberg, T. Fundamental understanding and modeling of reactive sputtering processes. Thin Solid Films 476, 215 (2005).

51. Kelly, P. \& Arnell, R. Magnetron sputtering: A review of recent developments and applications. Vacuum 56, 159 (2000).

52. Wolf, B. Handbook of Ion Sources. (1995). 
53. Jones, A. C. \& Hitchman, M. L. Chemical Vapour Deposition - Precursors, Processes and Applications. (Royal Society of Chemistry, 2008).

54. Schubert, U. \& Hüsing, N. Syhtesis of Inorganic Materials. (Wiley-VCH Verlag Gmbh \& Co. KGaA, Weinheim, 2005).

55. Hirako, A., Koiso, S. \& Ohkawa, K. Analysis of $\mathrm{TMGa} / \mathrm{NH}_{3} / \mathrm{H}_{2}$ reaction system in GaN-MOVPE growth by computational simulation. Phys. Status Solidi 203, 1716 (2006).

56. Chen, Q. \& Dapkus, P. D. On the Thermal Decomposition of Trimethylgallium A Molecular Beam Sampling Mass Spectroscopy Study. J. Electrochem. Soc. 138, 2821 (1991).

57. Hildebrandt, G. The Discovery of the Diffraction of X-rays in Crystals - A Historical Review. Cryst. Res. Technol. 28, 747 (1993).

58. Birkholz, M. Thin film analysis by X-ray scattering. (Wiley-VCH Verlag Gmbh \& Co. KGaA, Weinheim, 2006).

59. Williams, D. B. \& Carter, C. B. in Transmission electron microscopy (Springer Science+Buissnes Media, LLC, 1996).

60. Frängsmyr, T. \& Ekspång, G. Ernst Ruska - Biographical. (1993). at < "Ernst Ruska - Biographical”. Nobelprize.org. Nobel Media AB 2014. Web. 7 Jan 2015. <http://www.nobelprize.org/nobel_prizes/physics/laureates/1986/ruskabio.html > (Last accessed Dec 2104)

61. Veliadis, J. V. D., Khurgin, J. B., Ding, Y. J., Cui, A. G. \& Katzer, D. S. Investigation of the photoluminescence-linewidth broadening in periodic multiple narrow asymmetric coupled quantum wells. Phys. Rev. B 50, 4463 (1994).

62. Meeder, A., Marron, D. F., Chu, V., Conde, J. P., Jäger-Waldau, A., Rumberg, A., Luxsteiner, M. Ch. Photoluminescence and sub band gap absorption of CuGaSe 2 thin films. Thin Solid Films 404, 495 (2002). 


\section{Summary of Included Papers}

\section{Paper I}

In this paper, hybrid structures based on $\mathrm{AlGaN} / \mathrm{GaN}$ quantum well (QW) structures and colloidal $\mathrm{ZnO}$ nanocrystals (NC) have been studied by time-resolved photoluminescence (TRPL) measurements in the temperature range from 5-300 K. The $\mathrm{ZnO}$ nanocrystals were deposited on top of the QW structures having different cap layer thicknesses between 3 and $9 \mathrm{~nm}$. The quantum well exciton lifetime was extracted from PL decay curves detected for the bare QW structures and for the hybrids. The effect of $\mathrm{ZnO} \mathrm{NC}$ film on the QW exciton dynamics is strongly dependent on cap layer thickness. It is suggested that several recombination mechanisms with opposite effect can contribute to the QW exciton life time in hybrids including a non-radiative resonance energy transfer and variation of surface potential in the presence of $\mathrm{ZnO}$ NCs.

\section{Paper II}

In this paper, we study structural and optical properties of $\mathrm{GaN}$ nanorods grown on $\mathrm{Si}(111)$ substrates using a liquid Ga target in a DC magnetron sputtering system. Structural quality has been studied by X-ray diffraction and transmission electron microscopy (TEM). TEM images reveal a number of basal plane stacking faults (SF) having a characteristic signature at $3.42 \mathrm{eV}$ in luminescence spectra at 5 $\mathrm{K}$. Temperature dependent time-resolved photoluminescence show that the SFs related emission is stable until $290 \mathrm{~K}$, exhibiting at the same time a fast dynamics, which is typical for type I QWs. However a basal plane SF in wurtzite GaN forms a type II QW with no confinement for holes. It is suggested in accordance with TEM results that SFs form a regular structure similar to multiple QWs were a more efficient confinement for carriers can be achieved 



\section{Papers}

The articles associated with this thesis have been removed for copyright reasons. For more details about these see:

http://urn.kb.se/resolve?urn=urn:nbn:se:liu:diva-113741 